ESAIM: COCV 27 (2021) 49

https://doi.org/10.1051/cocv/2021041
ESAIM: Control, Optimisation and Calculus of Variations

www.esaim-cocv.org

\title{
A SEQUENTIAL QUADRATIC HAMILTONIAN SCHEME TO COMPUTE OPTIMAL RELAXED CONTROLS
}

\author{
M. Annunziato ${ }^{1}$ And A. Borzì ${ }^{2, *}$
}

\begin{abstract}
A new sequential quadratic Hamiltonian method for computing optimal relaxed controls for a class of optimal control problems governed by ordinary differential equations is presented. This iterative approach is based on the characterisation of optimal controls by means of the Pontryagin maximum principle in the framework of Young measures, and it belongs to the family of successive approximations schemes. The ability of the proposed optimisation framework to solve problems with regular and relaxed controls, including cases with oscillations and concentration effects, is demonstrated by results of numerical experiments. In all cases, the sequential quadratic Hamiltonian scheme appears robust and efficient, in agreement with convergence results of the theoretical investigation presented in this paper.
\end{abstract}

Mathematics Subject Classification. 49J15, 49K15, 49M05, 65K10.

Received May 22, 2020. Accepted April 6, 2021.

\section{INTRODUCTION}

Calculus of variation and optimal control theory have always provided a great stimulus for the development of new mathematical concepts with far reaching consequences, and among these ideas we can certainly point out the notion of generalized curves introduced by Young (later called Young measures) and the maximum principle in variational and control problems proposed by Pontryagin and his collaborators. The former concept considerably extends the meaning of solutions to variational and optimal control problems, and the latter represents a very general framework to characterize optimality.

We could say that, in his work, Young continued a tradition in the calculus of variation (and in all mathematical fields) to enlarge the space of solutions for those problems which do not admit a solution in a, say, ordinary space. This procedure is called relaxation and this is the reason why controls in Young's framework are called relaxed controls. Similarly, Pontryagin's approach to optimal control addresses the issue to characterize control functions, representing strong minima of a constrained minimisation problem, by means of needle variations thus introducing a larger variational space than previously thought. However, although the works of Young and Pontryagin have had a decisive impact in modern optimal control theory, their applicability has been restrained by the difficulty to construct robust and efficient numerical strategies. This statement may not be true for small-sized smooth control problems with ordinary differential equations (ODEs), but it is certainly

Keywords and phrases: Young measure, optimal relaxed controls, Pontryagin maximum principle, sequential quadratic Hamiltonian method, Kullback-Leibler divergence, numerical optimisation.

1 Dipartimento di Fisica "E. R. Caianiello", Università degli Studi di Salerno, Via G. Paolo II 132, 84084 Fisciano, Italy.

${ }^{2}$ Institut für Mathematik, Universität Würzburg, Emil-Fischer-Strasse 30, 97074 Würzburg, Germany.

* Corresponding author: alfio.borzi@mathematik.uni-wuerzburg.de 
true in case of optimal relaxed control problems and non-smooth large-sized regular (as opposed to relaxed) control problems.

For this purpose, in a series of recent works [5-7], a numerical optimisation scheme, called the sequential quadratic Hamiltonian (SQH) method, has been proposed and analysed that allows to efficiently solve nonsmooth (even discontinuous) large-sized optimal control problems in the framework of the Pontryagin maximum principle (PMP). It is the purpose of our work to investigate extensions of the SQH method to compute optimal relaxed controls in the framework of Young measures and PMP optimality; see [15] for a detailed discussion of both topics.

Therefore we would like to contribute to the field of numerical methods to solve relaxed optimal control problems where only few results are already available $[10,11,16,17,23,32]$. Specifically, in [10, 23] the moments approach is proposed focusing on a linear governing model and a $L^{1}$ cost functional. In this case, the linear model is approximated with Chebychev polynomials, and the resulting system of trajectories comes up to a simplified linear matrix inequality (LMI), where the unknown are the moments of the measures. In [11, 17], the focus is on problems with concentration, oscillations, and discontinuities, and a generalized DiPerna-Majda relaxed measure, i.e. anisotropic parametrized measure, that can be decomposed into a measure in time and a Young measure is used. In this framework, an occupation measure can be introduced, so that the problem is recast to a linear program over measure space, and the state equation and the cost functional can be non-linear and non-convex. If the problem data is polynomial or it can be put in this form, then the moments method is used.

An approach similar to ours can be found in [16,32], since it is formulated in the PMP framework and aims at defining a minimizing sequence of approximated measures for the value of the Hamilton-Pontryagin (HP) function. However, in these references, it is suggested to construct this sequence by a not clearly motivated linear combination strategy that is not explicitly formulated and the theoretical discussion seems limited to the case of a linear-affine problem.

In the next section, a short introduction to a class of optimal control problems is given, with the purpose to outline the argument for proving existence of regular optimal control and explain in which case the need of optimal relaxed controls may arise. Thus, the concept of Young measure is introduced, and the given class of optimal control problems is reformulated and discussed in this framework. In Section 3, the maximum principle is discussed in the regular and relaxed cases, and concluding with the formulation of the PMP optimality system. In Section 4, the development of successive approximations schemes is illustrated starting from the first approach by Krylov and Chernous'ko to the improvements by Sakawa and Shindo with the introduction of an augmented HP function. Then, these ideas are combined and further elaborated in the SQH method given in form of a pseudo algorithm. In this algorithm, we have a pointwise-in-time maximisation step of the augmented HP function and an adaptive step to update the weight of the augmented term to guarantee convergence as discussed in the following sections. In fact, the augmentation strategy is crucial for the efficiency and robustness of the SQH scheme, and we devote the Sections 5 and 6 to discuss this strategy focusing on the KullbackLeibler divergence. In Section 7, results of numerical experiments are presented that demonstrate the ability of the SQH scheme to solve optimal control problems with relaxed controls, including cases with oscillations and concentration effects. In Section 8, we discuss the case of relaxed control with a bioreactor model with two control mechanisms. A section of conclusion completes this work.

\section{Young MEASURES AND OPTIMAL RELAXED CONTROLS}

A large class of optimal control problems of interest in application has the following so-called Bolza structure (see, e.g., [4])

$$
\begin{aligned}
& \min J(y, u):=\int_{a}^{b} \ell(x, y(x), u(x)) \mathrm{d} x+\varphi(y(b)) \\
& \text { s.t. } \quad y^{\prime}(x)=f(x, y(x), u(x)), \quad y(a)=y_{a}, \\
& u \in U_{a d}
\end{aligned}
$$


(s.t. means 'subject to') where $J$ is called the cost functional, $\ell$ represents the running cost, $\varphi$ the terminal observation, and $f$ determines the dynamics of the governing system whose configuration is represented by the state variable $y$ driven by the control function $u$ chosen in the admissible set $U_{a d}$. We deal with the case where $y$ and $u$ are scalar functions; however, in a later section, we report results with a nonlinear system with a two-components state function and two controls.

In many cases, the running cost has a composite structure $\ell(x, y(x), u(x))=h(y(x))+g(u(x))$, where $h$ and $g$ are convex and usually differentiable, and the same is assumed for $\varphi$. Further, we assume that the function $f$ is continuous in $(x, y, u)$, Lipschitz-continuous in $y$, and for each fixed $y$ and $u \in U_{a d}, f(x, y, u(x))$ is measurable in $x$. We also require that, for any $c>0$, there exists a positive function $\gamma_{c} \in L^{1}(a, b)$ such that $|f(x, y, v)| \leq \gamma_{c}(x)$, for $x \in[a, b],|y| \leq c, v \in K_{a d}$; see below.

Subject to these conditions, the solution of the Cauchy problem in (2.1) admits a unique solution $y$ in the space of absolutely continuous functions in the interval $[a, b]$; see; e.g., [12] (Carathéodory theorem). Moreover, if there exists a continuous function $F$ of $y_{a}$ and $\|u\|_{U}$, such that the following estimate holds

$$
\left\|y^{\prime}\right\|_{L^{2}(a, b)} \leq F\left(\left|y_{a}\right|,\|u\|_{U}\right)
$$

then $y \in H:=H^{1}(a, b)$.

A specific choice of the admissible set of controls is the following closed, convex and bounded subset of the control space $U=L^{2}(a, b)$ given by

$$
U_{a d}=\left\{u \in L^{2}(a, b): u(x) \in K_{a d} \text { a.e. in }(a, b)\right\}
$$

where $K_{a d}$ denotes a compact subset of $\mathbb{R}$. However, if $g$ is coercive in $u$, then the optimal control problem above is well defined with $U_{a d}=U$.

In this setting, we have well-posedness of the control-to-state map

$$
S: L^{2}(a, b) \rightarrow H^{1}(a, b), \quad u \mapsto y=S(u),
$$

which provides the solution to the Cauchy problem in (2.1) for a given $u \in U$. Notice that, in most problems considered in application, the function $f$ is such that, for any weakly convergent sequence $\left(u^{k}\right) \subset U, u^{k} \rightarrow u$ in $U$, and assuming that the corresponding sequence $\left(y^{k}\right) \subset H, y^{k}=S\left(u^{k}\right)$, converges strongly in $C([a, b]$ ) (by embedding), $y^{k} \rightarrow y$, the weak limit $f\left(\cdot, y^{k}, u^{k}\right) \rightarrow f(\cdot, y, u)$ is well defined. Thus, one obtains that $y=S(u)$.

With this preparation and by the direct method of the calculus of variation [14], based on the construction of minimizing sequences, say $\left(u^{k}\right)$, one can prove existence of an optimal control $u \in U_{a d}$; see, e.g., [15]. In this proof, one is confronted with the problem of taking the limits of $f\left(\cdot, y^{k}, u^{k}\right)$ and $\ell\left(\cdot, y^{k}, u^{k}\right)$ where both functions $f$ and $\ell$ are usually nonlinear in their third argument. However, in the case of linear and linear-affine control mechanisms (in $f$ ), and $\ell$ convex such that $J$ is weakly lower semicontinuous, the limiting process is well defined and an optimal control where $J$ attains its minimum is obtained. We refer to this control as a regular optimal control.

In a more general situation, where $\ell$ is non-convex in $u$ and $f$ is nonlinear in $u$, the direct method above cannot be applied and a regular optimal control in $U_{a d}$ may not exist, although one can usually show that inf $J$ is finite.

Along the history of the development of the calculus of variation, we encounter many times the problem of nonexistence of solutions of optimisation problems that is resolved by enlarging the space of solutions. For example, searching for solutions in the space of piecewise $C^{1}$ functions (broken extremals) instead of continuous differentiable functions. This procedure, called relaxation, was also pursued by Young to address the situation illustrated above; see [34] and the references therein. A main result of his pioneering work is that given a sequence of measurable functions $z_{k}:[a, b] \rightarrow \mathbb{R}, k \in \mathbb{N}$, such that $\sup _{k} \int_{a}^{b} g\left(\left|z_{k}(x)\right|\right) \mathrm{d} x<\infty$, where $g$ is a continuous, non-decreasing function with $\lim _{t \rightarrow \infty} g(t)=\infty$, there exists a subsequence $\left(z_{m}\right)$ of $\left(z_{k}\right)$ and a family of regular 
probability measures $\left(\nu_{x}\right)$ on $\mathbb{R}$, such that the weak limit $\phi\left(z_{m}(\cdot), \cdot\right) \rightarrow \bar{\phi}(\cdot)$ is given by the expected value

$$
\bar{\phi}(x)=\int_{\mathbb{R}} \phi(v, x) d \nu_{x}(v), \quad \text { a.e. } x \in[a, b],
$$

for any Carathéodory function $\phi: \mathbb{R} \times[a, b] \rightarrow \mathbb{R}$. The family of probability measures $\left(\nu_{x}\right)$ is called the Young measure; see $[1,8,15,19,25,33,34]$ (an incomplete list) for more general definitions and much more details.

For our purpose, we would like to recall the comment in [1] stating that, intuitively, the Young measure can be thought of as giving the limiting probability distribution as $m \rightarrow \infty$ of the values of $z_{m}$ at almost all $x$. This intuition occurs clearly considering minimizing sequences for cost functionals (as the one discussed below) that are bounded from below but do not attain their minimum. Moreover, one can see by construction that the functions $z_{m}$ become increasingly oscillatory as $m \rightarrow \infty$; see [33,34]. On the other hand, one can interpret a Young measure associated to a measurable function as the unique measure concentrated on its graph. In particular, we may identify a regular optimal control $u$ as the family of measures with $\nu_{x}(v)=\delta(v-u(x))$ on $[a, b]$, where $\delta$ denotes the Dirac delta. In general, notice that $\nu_{x}$ is a probability measure so that $\nu_{x}(v) \geq 0$, $v \in K_{a d}$ and $\nu_{x}\left(K_{a d}\right)=1$ almost everywhere in $[a, b]$; see [15]. We refer to $\nu_{(\cdot)}$ as a relaxed control.

In the framework of Young measures, a relaxed version of the optimal control problem (2.1) is formulated as follows [15]:

$$
\begin{aligned}
& \min J(y, \nu):=\int_{a}^{b} \int_{K_{a d}} \ell(x, y(x), v) \mathrm{d} \nu_{x}(v) \mathrm{d} x+\varphi(y(b)) \\
& \text { s.t. } \quad y^{\prime}(x)=\int_{K_{a d}} f(x, y(x), v) \mathrm{d} \nu_{x}(v), \quad y(a)=y_{a} .
\end{aligned}
$$

In this framework, the control set $\mathcal{K}_{a d}$ is the space of probability measures defined in the dual of $C\left(K_{a d}\right)$, and the admissible control space $\mathcal{U}_{a d}$ is the space of relaxed controls consisting of all probability-measure valued $\nu_{(\cdot)}$ in $L^{1}\left(a, b ; C\left(K_{a d}\right)\right)^{*}$.

We refer to [15] for a proof of the Relaxation Theorem stating that for $\nu_{(\cdot)} \in \mathcal{U}_{a d}$ such that the Cauchy problem in (2.3) has a solution $y_{\nu}$ in $[a, b]$, there exists a corresponding piecewise constant function $u$ such that the corresponding solution $y_{u}$ of the Cauchy problem in (2.1) exists in $[a, b]$ and approximates (pointwise) $y_{\nu}$ to any desired accuracy $\epsilon>0$, i.e. $\left|y_{\nu}(x)-y_{u}(x)\right|<\epsilon, x \in[a, b]$.

Next, assume that $\ell(x, y, u)$ is measurable in $x$ for $y$ and $u$ fixed, and continuous in $y$ and $u$ for $x$ fixed. Further, suppose that for every $c>0$ there exists a function $\Gamma_{c} \in L^{1}(a, b)$ such that

$$
|\ell(x, y, v)| \leq \Gamma_{c}(x), \quad x \in[a, b],|y| \leq c, v \in K_{a d} .
$$

Then, we have that $J(y, \nu)$ is weakly lower semicontinuous in the space of relaxed controls [15]. Also in this reference, a proof is given that, with the setting above, there exists an optimal relaxed control $\nu_{(\cdot)} \in \mathcal{U}_{a d}$. This proof is based on the direct method of the calculus of variation that involves minimizing sequences $\nu_{(\cdot)}^{n} \rightarrow \nu_{(\cdot)}$, and the fact that the space of relaxed controls is compact in the weak-star topology. Notice that, in general, for the class of optimal control problems considered above, uniqueness of optimal (relaxed) controls cannot be established.

\section{The MAXIMUM PRINCIPLE}

Since the work of Pontryagin and his collaborators Boltyanskiu and Gamkrelidze $[2,26]$, the so-called maximum principle in optimal control is well known. This principle establishes a necessary condition of optimality of the control in (2.1) based on the following Hamilton-Pontryagin (HP) function

$$
H(x, y, p, u)=p f(x, y, u)-\ell(x, y, u) .
$$


Let us notice that using this function, our governing model can be re-formulated as follows

$$
\begin{aligned}
y^{\prime}(x) & =\frac{\partial H}{\partial p}(x, y(x), p(x), u(x)), \\
y(a) & =y_{a} .
\end{aligned}
$$

In this formulation, the function $p$ represents the adjoint variable (Lagrange multiplier), which is given by the solution of the following adjoint Cauchy problem [4]

$$
\begin{aligned}
p^{\prime}(x) & =-\frac{\partial H}{\partial y}(x, y(x), p(x), u(x)), \\
p(b) & =-\frac{\partial \varphi}{\partial y}(y(b)) .
\end{aligned}
$$

Notice that this last equation requires differentiability of $f$ and $\ell$ with respect to $y$, which we assume.

Now, let us denote with $u^{*} \in U_{a d}$ an (regular) optimal control for (2.1). Correspondingly, we have $y^{*}=S\left(u^{*}\right)$, and $p^{*}$ is given by (3.3) with $u=u^{*}$ and $y=y^{*}$. In the framework of the Pontryagin maximum principle (PMP), the characterisation of optimality of $\left(y^{*}, p^{*}, u^{*}\right)$ is formulated as follows

$$
H\left(x, y^{*}(x), p^{*}(x), u^{*}(x)\right) \geq H\left(x, y^{*}(x), p^{*}(x), v\right), \quad v \in K_{a d},
$$

for almost all $x \in[a, b]$. This means that along the optimal triple $\left(y^{*}, p^{*}, u^{*}\right)$ and almost all $x$ fixed, $H\left(x, y^{*}(x), p^{*}(x), v\right)$ attains a maximum at $v=u^{*}(x)$.

Clearly, assuming that $f$ and $\ell$, and thus $H$, are differentiable with respect to $u$, this characterisation implies that

$$
\frac{\partial H}{\partial u}\left(x, y^{*}(x), p^{*}(x), u^{*}(x)\right)\left(v-u^{*}(x)\right) \leq 0, \quad v \in K_{a d},
$$

for almost all $x \in[a, b]$.

The maximum principle has a counterpart in the framework of relaxed controls; see, e.g., [15, 28, 33]. For its formulation in this case, we assume the following:

Assumption 3.1. The functions $f(x, y, u)$ and $\ell(x, y, u)$ are continuously differentiable in $y$, the partial derivative $\partial_{y} f(x, y, u)$ and $\partial_{y} \ell(x, y, u)$ are measurable in $x$ for $y$ and $u$ fixed, and continuous in $y$ and $u$ for $x$ fixed. Moreover, for every $c_{1}, c_{2}>0$ there exist functions $\beta_{c_{1}}, \beta_{c_{2}} \in L^{1}(a, b)$ such that

$$
\left|\partial_{y} f(x, y, v)\right| \leq \beta_{c_{1}}(x), \quad x \in[a, b],|y| \leq c_{1}, v \in K_{a d}
$$

and

$$
\left|\partial_{y} \ell(x, y, v)\right| \leq \beta_{c_{2}}(x), \quad x \in[a, b],|y| \leq c_{2}, v \in K_{a d}
$$

Next, we write the relaxed HP function

$$
\mathcal{H}(x, y, p, \mu)=p \int_{K_{a d}} f(x, y, v) \mathrm{d} \mu(v)-\int_{K_{a d}} \ell(x, y, v) \mathrm{d} \mu(v)
$$

where $\mu \in \mathcal{K}_{a d}$. 
Correspondingly, the PMP optimality conditions for (2.3) are given by

$$
\begin{aligned}
& y^{\prime}(x)=\frac{\partial \mathcal{H}}{\partial p}\left(x, y(x), p(x), \nu_{x}\right), \quad y(a)=y_{a}, \\
& p^{\prime}(x)=-\frac{\partial \mathcal{H}}{\partial y}\left(x, y(x), p(x), \nu_{x}\right), \quad p(b)=-\frac{\partial \varphi}{\partial y}(y(b)), \\
& \mathcal{H}\left(x, y(x), p(x), \nu_{x}\right)=\max _{\mu \in \mathcal{K}_{a d}} \mathcal{H}(x, y(x), p(x), \mu) \quad \text { a. e. in }[a, b] .
\end{aligned}
$$

If $\left(y^{*}, p^{*}, \nu^{*}\right)$ is a solution of the relaxed optimal control problem, then this triple must satisfy these PMP conditions.

Since the work of Young [34], the construction of optimal relaxed controls is usually illustrated by the following problem.

Problem 3.2. Consider

$$
f(x, y, u)=u, \quad \ell(x, y, u)=y^{2}+\left(u^{2}-1\right)^{2}, \quad \varphi \equiv 0
$$

and $K_{a d}=[-1,1]$. One can verify that the conditions on $f, \ell$ and $\varphi$ mentioned above are satisfied and thus an optimal relaxed control exists; see [15]. Choosing $y(a)=0$, the resulting optimal relaxed control is given by the measure

$$
\nu_{x}^{*}(v)=\frac{1}{2} \delta(v-1)+\frac{1}{2} \delta(v+1)
$$

and $y^{*}(x)=0, p^{*}(x)=0, x \in[a, b]$.

We would like to remark that the same solution is obtained if we enlarge the control set such that $K_{a d} \supset$ $[-1,1]$. The same is true if we consider our problem in a non-compact control set as, e.g., $K_{a d}=\mathbb{R}$. This fact appears from the PMP in the maximisation of the (relaxed) HP function at optimality as follows

$$
\int_{K_{a d}}\left(v^{2}-1\right)^{2} d \nu_{x}^{*}(v)=\min _{\mu \in \mathcal{K}_{a d}} \int_{K_{a d}}\left(v^{2}-1\right)^{2} \mathrm{~d} \mu(v) .
$$

Notice that, apart of this and similar problems, it is very difficult to determine an optimal relaxed control explicitly, and a numerical procedure that allows to compute approximations to relaxed optimal controls is required.

\section{SuCCESSIVE APPROXIMATIONS SCHEMES}

The first version of the method of successive approximations (SA) for solving optimal control problems as (2.1) appeared in [20] soon after the formulation of the maximum principle. This method consists of successive integrations of the governing model (3.2), then of the adjoint problem (3.3), followed by an update of the control by maximisation of the HP function as given in (3.4). An early review of variants of the SA scheme can be found in [9]. However, as discusses in these earlier references, the proposed SA schemes appear less robust with respect to the choice of initialisation and of the value of weight of the cost of the control. For this reason, in [21], different variants of the SA method are discussed that transform the state equation to obtain a weakly controlled problem or use a damping of the control update, or restrict the change of the control to a short time window. Clearly, these techniques are less satisfactory, and it is with the adaptive quadratic penalisation of the HP function proposed in $[29,30]$ that a robust SA procedure is obtained. On the other hand, although convergence of this new procedure could be proved [3,29,30], the requirement of a frequent update of the state variable, during the update of the control step, has limited the range of application of this method to small 
size problems. However, this limitation has been removed in a series of recent works [5-7], thus making possible to successfully apply the resulting SA scheme to control problems governed by partial differential equations. This new variant of the SA scheme, called the sequential quadratic Hamiltonian (SQH) method, combines the advantageous computational complexity of the method in [20] with the robustness of the SA variant in [29, 30]. As in these earlier works, the iterative SQH scheme considers the following augmented HP function

$$
H_{\epsilon}(x, y, p, v, u):=H(x, y, p, u)-\epsilon(u-v)^{2},
$$

where $\epsilon>0$ is a variable penalisation parameter, and $v$ plays the role of the approximation to the control function obtained at the preceding iteration. We remark that the idea of adding a quadratic penalisation to a function to be iteratively optimised as in (4.1) could be traced back to the weighted Jacobi iteration in numerical linear algebra. Sakawa and Shindo [29] relate their approach to the work of B. Järmark in 1975 [18], which anticipates the proximal scheme proposed by R. T. Rockafellar in [27]. Notice that, in all these works, differentiability of $H$ with respect to $u$ (in a convex set) is required, which is not the case in our SQH approach.

It is clear that existing SA methods cannot be applied to problems whose solutions can only be found in the framework of relaxed controls as Problem 3.2. On the other hand, based on the formulation of the PMP for Young measures, we propose to extend the SA strategy to compute optimal relaxed controls. For this purpose, we focus on a new version of the SQH scheme that is implemented in the following (pseudo) algorithm $(z \leftarrow w$ means update the value of $z$ with the value of $w$ ).

Algorithm 4.1 (SQH method).

1. Choose $\epsilon>0, \kappa>0, \sigma>1, \zeta \in(0,1), \eta \in(0, \infty), \nu^{0} \in \mathcal{U}_{a d}$, compute $y^{0}$ by integrating (3.5) for $\nu=\nu^{0}$, and $p^{0}$ by solving (3.6) with $y=y^{0}$ and $\nu=\nu^{0}$, set $k=0$.

2. Find $\nu \in \mathcal{U}_{a d}$ such that

$$
\mathcal{H}_{\epsilon}\left(x, y^{k}(x), p^{k}(x), \nu_{x}^{k}, \nu_{x}\right)=\max _{\mu \in \mathcal{K}_{a d}} \mathcal{H}_{\epsilon}\left(x, y^{k}(x), p^{k}(x), \nu_{x}^{k}, \mu\right)
$$

for almost all $x \in[a, b]$.

3. Calculate $y$ by integrating (3.5) with $\nu$ given by Step 2, and set $\tau:=\left\|\nu-\nu^{k}\right\|_{L^{1}\left([a, b] \times K_{a d}\right)}$

4. If $J(y, \nu)-J\left(y^{k}, \nu^{k}\right)>-\eta \tau$ :

Choose $\epsilon \leftarrow \sigma \epsilon$

If max of $\epsilon$ : return $\nu^{k}$ and STOP with warning

Else: go to 2

Else:

Choose $\epsilon \leftarrow \zeta \epsilon$, set $y^{k+1}=y, \nu^{k+1}=\nu$, calculate $p^{k+1}$ by integrating (3.6)

with $y=y^{k+1}$ and $\nu=\nu^{k+1}$, set $k \leftarrow k+1$

5. If $\tau<\kappa$ : STOP and return $\nu^{k}$

Else: go to 2.

Our main working hypothesis is that the optimal relaxed control is absolutely continuous such that we can express the probability measure sought in terms of a probability density function (PDF). However, for simplicity, we use the same symbols and write $\mathrm{d} \nu_{x}(v)=\nu(v, x) \mathrm{d} v$. Further, we assume that $\mu, \nu \in C^{1}\left([a, b], L^{2}\left(K_{a d}\right)\right)$, and focus on the following augmented HP function

$$
\begin{gathered}
\mathcal{H}_{\epsilon}(x, y(x), p(x), \mu(\cdot, x), \nu(\cdot, x))=p(x) \int_{K_{a d}} f(x, y(x), v) \nu(v, x) \mathrm{d} v \\
-\int_{K_{a d}} \ell(x, y(x), v) \nu(v, x) \mathrm{d} v-\epsilon g(\nu(\cdot, x), \mu(\cdot, x)) .
\end{gathered}
$$


Notice that the functions $y$ and $p$ are related to the density $\mu$.

In the next section, we discuss different choices of the augmenting function $g$ and, correspondingly, different numerical schemes for implementing Step 2 in Algorithm 4.1.

\section{Augmentation and optimisation}

The focus of this section is the investigation of numerical procedures for the realisation of Step 2 of the SQH Algorithm 4.1, where $\mathcal{H}_{\epsilon}$ is given in (4.2) with an augmentation function $g$ to be chosen appropriately. In fact, in the spirit of the quadratic term in (4.1), we require that the function $g$ be convex in its first argument.

First, we discuss an extension of (4.1) by choosing

$$
g(\nu(\cdot, x), \mu(\cdot, x))=\frac{1}{2} \int_{K_{a d}}|\nu(v, x)-\mu(v, x)|^{2} \mathrm{~d} v
$$

Notice that, for $x$ fixed, $\mathcal{H}_{\epsilon}$ is a quadratic (concave) functional of $\nu(\cdot, x)$. Clearly, this functional is Fréchet differentiable in $L^{2}\left(K_{a d}\right)$ with scalar product $(\cdot, \cdot)$. In this space, we can introduce the gradient of $\mathcal{H}_{\epsilon}$ with respect to $\nu$ as follows (to simplify our discussion, we omit to write $x, y(x)$, and $p(x)$, which are assumed fixed). We have

$$
\left(\nabla \mathcal{H}_{\epsilon}(\nu), \delta \nu\right)=\int_{K_{a d}}[H(v)-\epsilon(\nu-\mu)] \delta \nu(v) \mathrm{d} v
$$

where $H(v):=H(x, y(x), p(x), v)=p(x) f(x, y(x), v)-\ell(x, y(x), v)$.

The availability of $\nabla \mathcal{H}_{\epsilon}(\nu):=H(v)-\epsilon(\nu-\mu)$ suggests the construction of the following projected-gradient iterative procedure

$$
\nu^{(m+1)}=\mathcal{P}\left(\nu^{(m)}+\lambda \nabla \mathcal{H}_{\epsilon}\left(\nu^{(m)}\right)\right), \quad \nu^{(0)}=\mu,
$$

where $m=0,1,2, \ldots$, and $\lambda>0$ is a chosen step size. However, taking $\lambda=1 / \epsilon$, and considering only one step, we have

$$
\nu(v, x)=\mathcal{P}(\mu(v, x)+H(v, x) / \epsilon) .
$$

Notice that $\mathcal{P}$ is an operator that takes the positive part of its argument and normalizes the resulting function with respect to its $L^{1}\left(K_{a d}\right)$-norm. In fact, the formula (5.3) gives the solution to the gradient equation subject to the aforementioned conditions.

Now, we present results obtained with Algorithm 4.1 applied to Problem 3.2, but choosing a non-zero initial condition, where Step 2 implements the optimisation scheme given in (5.3). In this computation, we choose $K_{a d}=[-2,2]$, where we consider a uniform grid with subintervals of size $h=0.01$, and on this grid we define the initial $\nu^{0}$ to be a uniform PDF. In the SQH scheme, we set $\epsilon=100, \sigma=1.1, \zeta=0.9, \eta=10^{-3}$, and $\kappa=10^{-4}$. The initial condition is $y(0)=y_{0}=1 / 2$.

The resulting optimal relaxed control obtained by the SQH scheme is depicted in Figure 1. This solution is obtained after a total of 391 iterations where the stopping criteria is met. Notice that the SQH scheme produces iterates that monotonically reduce the value of the relaxed cost functional; see Figure 2. (The value of $J$ at convergence is 0.04254 ).

We remark that the iterative procedure (5.3) is open to criticism because of the action of the operator $\mathcal{P}$ and the fact that $g$ given by (5.1) is not weakly* continuous. For this reason, we consider the well-known 

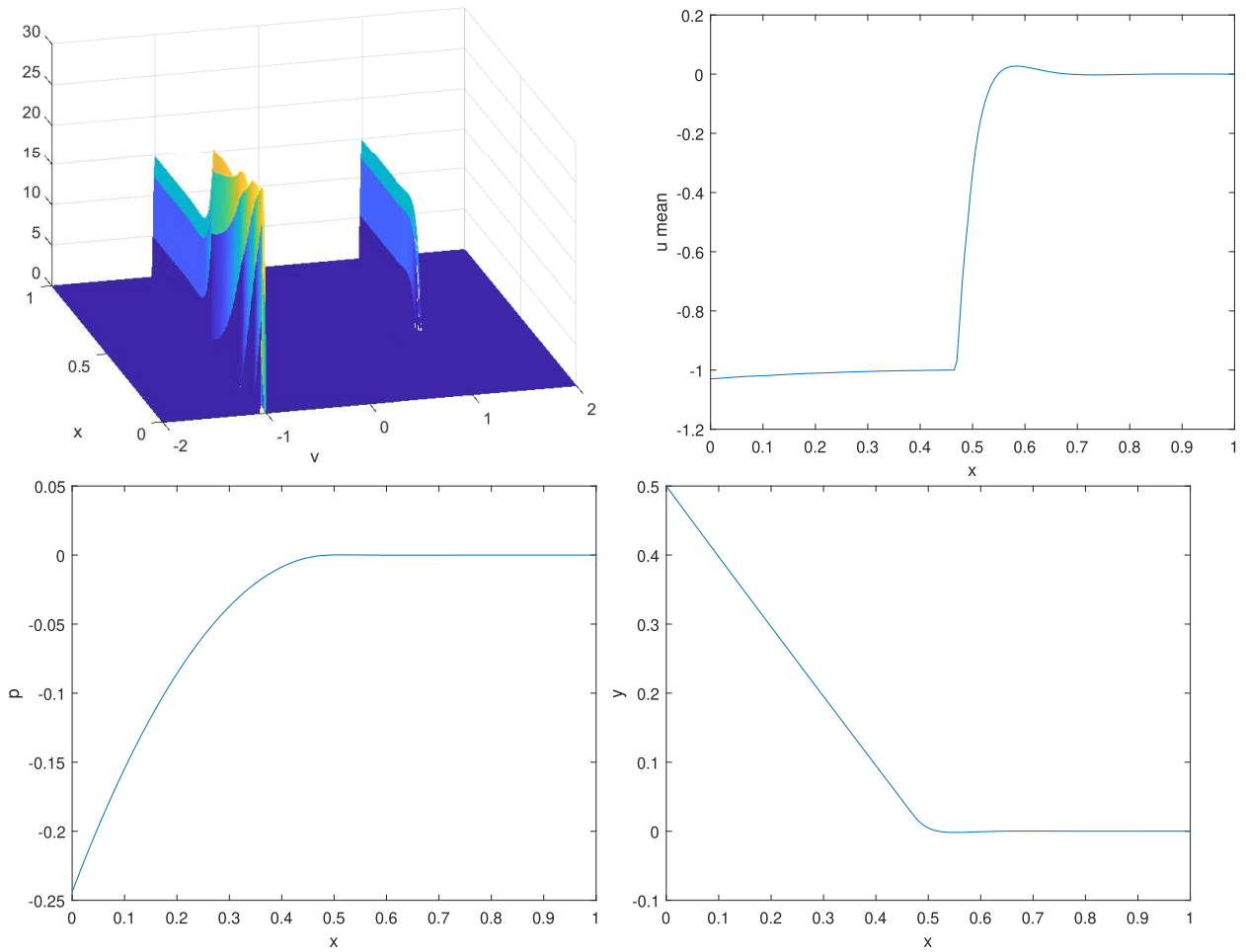

FiguRE 1. Solution of Problem 3.2 with $y_{0}=1 / 2$ obtained with Algorithm 4.1 and (5.3): from top-left to bottom-right, the optimal relaxed control, the mean of the optimal relaxed control, the adjoint variable, and the controlled state.

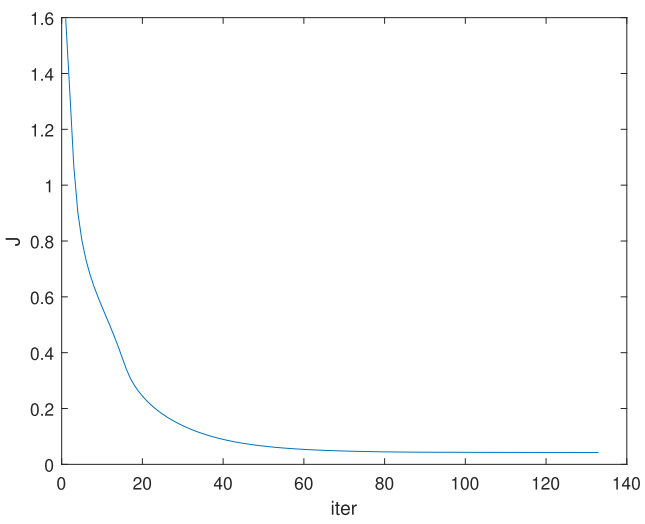

Figure 2. Convergence history of $J$ with Algorithm 4.1 and (5.3) for solving Problem 3.2 with $y_{0}=1 / 2$.

Kullback-Leibler (KL) divergence given by [22]

$$
D_{x}(\nu \| \mu):=\int_{K_{a d}} \nu(v, x) \log (\nu(v, x) / \mu(v, x)) \mathrm{d} v,
$$


where $\mu, \nu \in C^{1}\left([a, b], L^{1}\left(K_{a d}\right)\right)$; see also [13]. Thus, we investigate the following choice

$$
g(\nu(\cdot, x), \mu(\cdot, x))=D_{x}(\nu \| \mu) .
$$

In this case, for each fixed $x$ that we omit, we have

$$
\left(\nabla \mathcal{H}_{\epsilon}(\nu), \delta \nu\right)=\int_{K_{a d}}[H(v)-\epsilon(\log (\nu(v) / \mu(v))+1)] \delta \nu(v) \mathrm{d} v
$$

and

$$
\left(\nabla^{2} \mathcal{H}_{\epsilon}(\nu) \delta \nu, \delta \nu\right)=-\epsilon \int_{K_{a d}} \frac{\delta \nu(v)^{2}}{\nu(v)} \mathrm{d} v \leq 0
$$

Clearly, we can use the gradient (5.6) and define a new variant of (5.3), which will require to use the operator $\mathcal{P}$. However, this new setting allows to follow an alternative route, that is, we obtain $\nu$ from the given $\mu$ requiring that it solves the gradient equation $\nabla \mathcal{H}_{\epsilon}(\nu)=0$. Thus, we have $H(v, x) / \epsilon-1=\log (\nu(v, x) / \mu(v, x))$. Based on this result, we define the update in Step 2 of the SQH algorithm 4.1. We have

$$
\nu(v, x)=C(x) \mu(v, x) \exp (H(x, y(x), p(x), v) / \epsilon)
$$

where

$$
C(x)=1 / \mathbb{E}_{\mu(\cdot, x)}[\exp (H(x, y(x), p(x), \cdot) / \epsilon)] .
$$

In this statement, we have introduced the following notation

$$
\mathbb{E}_{\mu(\cdot, x)}[\phi(\cdot, x)]:=\int_{K_{a d}} \phi(v, x) \mu(v, x) \mathrm{d} v
$$

which represents the expectation of $\phi$ with respect to the density $\mu$ and for a fixed $x$.

Notice that in this case the update of the Young measure is automatically non-negative. Further notice that the Hessian (5.7) is well defined and negative, thus guaranteeing concavity. However, the update (5.8) requires normalisation that is encoded in the positive factor $C(x)$.

Next, we present results obtained with Algorithm 4.1 applied to Problem 3.2 with $y_{0}=1 / 2$, where Step 2 implements the optimisation scheme given in (5.8) with $\mu=\nu^{k}$ for each fixed $x$. In this experiment, we use the same setting as above. In Figure 3, we report results of this experiment showing the computed optimal relaxed control and its mean for all $x$ and, correspondingly, the state and the adjoint variables.

The relaxed optimal control solution is obtained after 448 iterations where the stopping criteria is met. The minimizing process of the SQH procedure is shown in Figure 4. (The value of $J$ at convergence is 0.04238.) We see that similar results to those of the previous experiment are obtained. However, the approach based on the KL divergence achieves a lower value of $J$. Further results and additional comments are postponed to the section on numerical experiments, where we focus on Algorithm 4.1 and (5.8) for solving different control problems.

\section{The SQH MinimizING PROPERTY}

In this section, we continue our discussion on the convergence properties of the SQH algorithm for solving (2.3). Our purpose is to prove that there exists a $\epsilon^{*}$ such that, for $\epsilon>\epsilon^{*}$, the Step 2 in the SQH algorithm results in an update of $\nu$ that satisfies the descent criteria for the functional $J$ given in Step 4. of this algorithm. This analysis is similar, in spirit, to that presented in, e.g., [7] and the references therein. However, in the present case 

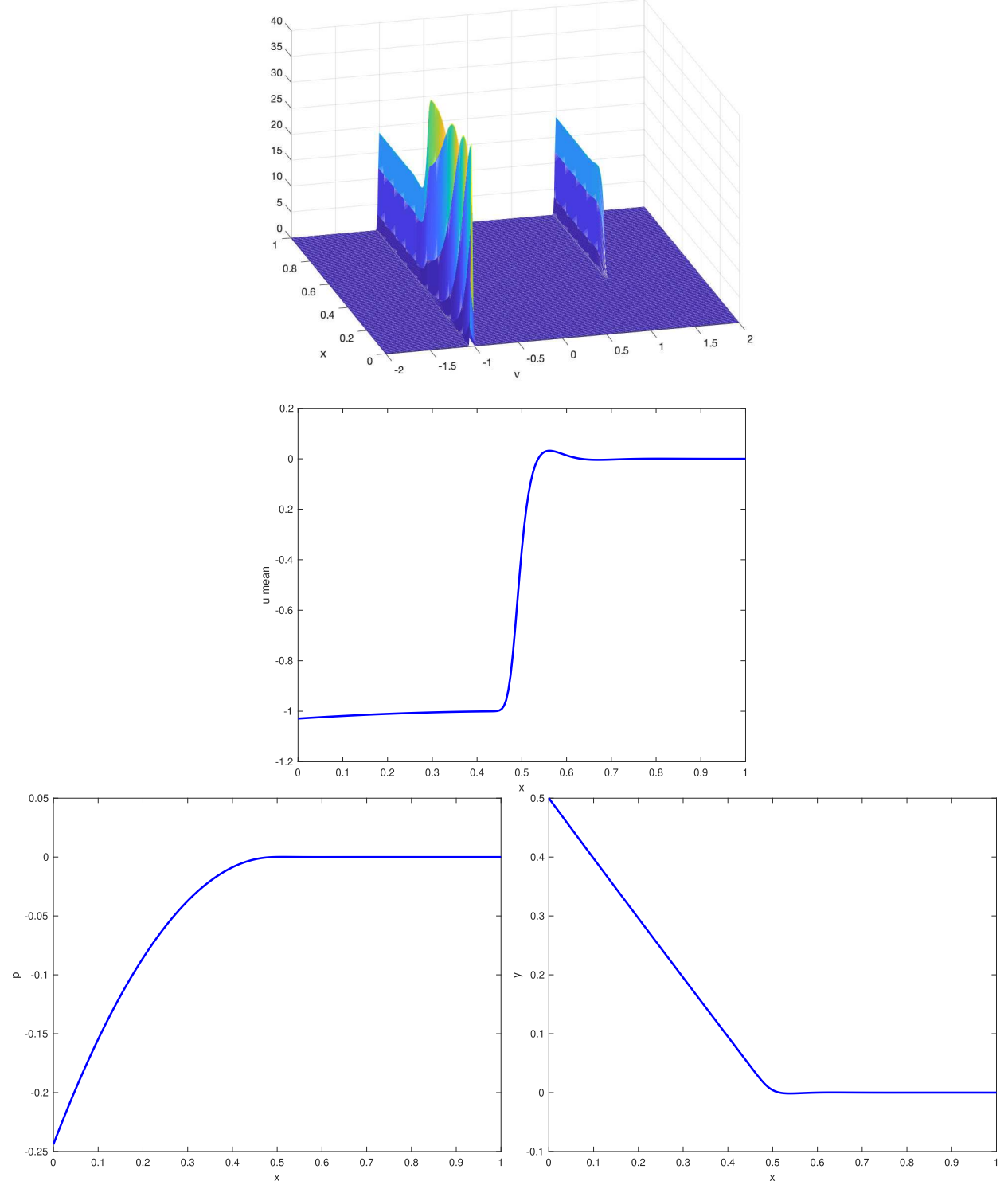

Figure 3. Solution of Problem 3.2 with $y_{0}=1 / 2$ computed with Algorithm 4.1 and (5.8): from top-left to bottom-right, the optimal relaxed control, the mean of the optimal relaxed control, the adjoint variable, and the controlled state.

the analysis is performed in the framework of Young measures, it includes the presence of a terminal term in the cost functional, and specifically accommodates the choice of the Kullback-Leibler divergence as augmenting term.

We make the following assumption:

Assumption 6.1. The functions $f(x, y, v)$ and $\ell(x, y, v)$ are twice continuously differentiable in $y$, the partial derivative $\partial_{y}^{2} f(x, y, u)$ and $\partial_{y}^{2} \ell(x, y, u)$ are measurable in $x$ for $y$ and $u$ fixed, and continuous in $y$ and $u$ for $x$ fixed. 


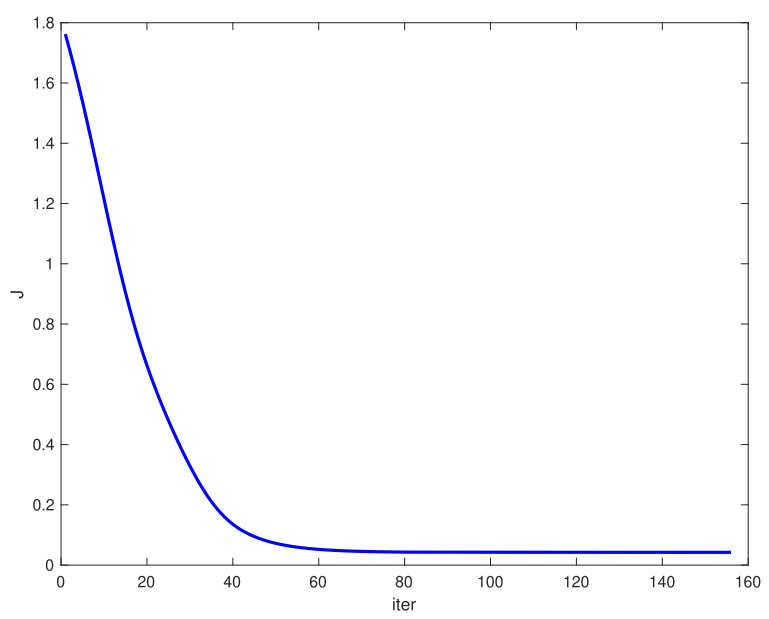

Figure 4. Convergence history of $J$ with Algorithm 4.1 and (5.8) for solving Problem 3.2 with $y_{0}=1 / 2$.

Theorem 6.2. Let $\nu^{k}$ a given density and $\nu^{\epsilon}$ the density as established in (5.8) with $\mu=\nu^{k}$; then, subject to the Assumptions 3.1 and 6.1 for the functions $f(x, y, v)$ and $\ell(x, y, v)$, there exists a value $\epsilon^{*}$ such that for $\epsilon>\epsilon^{*}$ it holds that $J\left(y^{\epsilon}, \nu^{\epsilon}\right)<J\left(y^{k}, \nu^{k}\right)$.

Proof. We define the functions difference $\delta y:=y^{\epsilon}-y^{k}, \delta \nu:=\nu^{\epsilon}-\nu^{k}, \delta p:=p^{\epsilon}-p^{k}$, where the index $\epsilon$ denotes functions computed in correspondence to $\nu^{\epsilon}$. For the Taylor expansion remainder terms that we use below, we introduce the functions $y_{\xi}, y_{\eta}, y_{\zeta}, y_{\omega}$ such that their values satisfy $y_{\xi}(x), y_{\eta}(x), y_{\zeta}(x), y_{\omega}(x) \in$ $\left[\min \left(y^{k}(x), y^{\epsilon}(x)\right), \max \left(y^{k}(x), y^{\epsilon}(x)\right)\right]$.

In the following, we use the notation:

$\mathbb{E}_{k}\left[f\left(x, y^{k}, \cdot\right)\right]=\int_{K_{a d}} f\left(x, y^{k}(x), v\right) \nu^{k}(v, x) \mathrm{d} v$, and

$\mathbb{E}_{\epsilon}\left[f\left(x, y^{k}, \cdot\right)\right]=\int_{K_{a d}} f\left(x, y^{k}(x), v\right) \nu^{\epsilon}(v, x) \mathrm{d} v$.

By construction, we have

$$
-\mathcal{H}_{\epsilon}\left(x, y^{k}(x), p^{k}(x), \nu^{k}(\cdot, x), \nu^{\epsilon}(\cdot, x)\right)+\mathcal{H}\left(x, y^{k}(x), p^{k}(x), \nu^{k}(\cdot, x)\right) \leq 0
$$

By expanding the terms in the Hamiltonian, we have

$$
\begin{aligned}
& \left.\left.p^{k} \mathbb{E}_{k}\left[f\left(x, y^{k}, \cdot\right)\right]-\mathbb{E}_{k}\left[\ell\left(x, y^{k}, \cdot\right)\right)\right]-p^{k} \mathbb{E}_{\epsilon}\left[f\left(x, y^{k}, \cdot\right)\right]+\mathbb{E}_{\epsilon}\left[\ell\left(x, y^{k}, \cdot\right)\right)\right] \\
& +\epsilon D_{x}\left(\nu^{\epsilon} \| \nu^{k}\right) \\
& \left.=p^{k} y^{k \prime}-\mathbb{E}_{k}\left[\ell\left(x, y^{k}, \cdot\right)\right)\right]-p^{k} \mathbb{E}_{\epsilon}\left[f\left(x, y^{k}, \cdot\right)\right]+\mathbb{E}_{\epsilon}\left[\ell\left(x, y^{\epsilon}-\delta y, \cdot\right)\right] \\
& +\epsilon D_{x}\left(\nu^{\epsilon} \| \nu^{k}\right) \\
& \left.\left.=\mathbb{E}_{\epsilon}\left[\ell\left(x, y^{\epsilon}, \cdot\right)\right)\right]-\mathbb{E}_{k}\left[\ell\left(x, y^{k}, \cdot\right)\right)\right]+p^{k} y^{k \prime}-\delta y \mathbb{E}_{\epsilon}\left[\left.\frac{\partial \ell}{\partial y}\right|_{y^{\epsilon}}\right]-p^{k} \mathbb{E}_{\epsilon}\left[f\left(x, y^{k}, \cdot\right)\right] \\
& +\frac{\delta y^{2}}{2} \mathbb{E}_{\epsilon}\left[\left.\frac{\partial^{2} \ell}{\partial y^{2}}\right|_{y_{\xi}}\right]+\epsilon D_{x}\left(\nu^{\epsilon} \| \nu^{k}\right) .
\end{aligned}
$$


We have used the state equation to obtain the derivative $y^{k \prime}$, and expanded $\ell\left(x, y^{\epsilon}-\delta y, v\right)$; the term $\left.\frac{\partial^{2} \ell}{\partial y^{2}}\right|_{y_{\xi}}$ represents the second derivative of $\ell$ with respect to $y$ evaluated to a certain unknown function $y_{\xi}$ for the Taylor's remainder.

Now, notice that the integral in $(a, b)$ of the first two terms in (6.1) corresponds to the difference of the running costs in the functionals $J\left(y^{\epsilon}, \nu^{\epsilon}\right)$ and $J\left(y^{k}, \nu^{k}\right)$ without the terminal costs $\varphi$.

Now, we proceed recasting the integral of the fourth addend in (6.1) as follows

$$
-\int_{a}^{b} \delta y(x) \mathbb{E}_{\epsilon}\left[\left.\frac{\partial \ell}{\partial y}\right|_{y^{\epsilon}}\right] \mathrm{d} x=-\int_{a}^{b} \delta y(x) p^{\epsilon}(x) \mathbb{E}_{\epsilon}\left[\left.\frac{\partial f}{\partial y}\right|_{y^{\epsilon}}\right] \mathrm{d} x-\int_{a}^{b} p^{\epsilon \prime}(x) \delta y(x) \mathrm{d} x
$$

where we have used the adjoint equation. Similarly, for the integral of the fifth addend we have

$$
\begin{aligned}
& -\int_{a}^{b} p^{k}(x) \mathbb{E}_{\epsilon}\left[f\left(x, y^{k}, \cdot\right)\right] \mathrm{d} x=-\int_{a}^{b} p^{k}(x) \mathbb{E}_{\epsilon}\left[f\left(x, y^{\epsilon}-\delta y, \cdot\right)\right] \mathrm{d} x \\
& =-\int_{a}^{b} p^{k}(x) \mathbb{E}_{\epsilon}\left[f\left(x, y^{\epsilon}, \cdot\right)\right] \mathrm{d} x+\int_{a}^{b} \delta y(x) p^{k}(x) \mathbb{E}_{\epsilon}\left[\left.\frac{\partial f}{\partial y}\right|_{y^{\epsilon}}\right] \mathrm{d} x \\
& +\int_{a}^{b} \frac{\delta y(x)^{2}}{2} p^{k}(x) \mathbb{E}_{\epsilon}\left[\left.\frac{\partial^{2} f}{\partial y^{2}}\right|_{y_{\eta}}\right] \mathrm{d} x=-\int_{a}^{b} p^{k}(x) y^{\epsilon \prime}(x) \mathrm{d} x \\
& +\int_{a}^{b} \delta y(x) p^{k}(x) \mathbb{E}_{\epsilon}\left[\left.\frac{\partial f}{\partial y}\right|_{y^{\epsilon}}\right] \mathrm{d} x+\int_{a}^{b} \frac{\delta y(x)^{2}}{2} p^{k}(x) \mathbb{E}_{\epsilon}\left[\left.\frac{\partial^{2} f}{\partial y^{2}}\right|_{y_{\eta}}\right] \mathrm{d} x,
\end{aligned}
$$

where we have used the state equation and integration by parts, and the second derivative is evaluated on the function $y_{\eta}$.

By summing up the last result with the third addend of (6.1), we obtain

$$
\begin{aligned}
& -\int_{a}^{b} p^{k}(x) \delta y^{\prime}(x) \mathrm{d} x+\int_{a}^{b} \delta y(x) p^{k}(x) \mathbb{E}_{\epsilon}\left[\left.\frac{\partial f}{\partial y}\right|_{y^{\epsilon}}\right] \mathrm{d} x+\int_{a}^{b} \frac{\delta y(x)^{2}}{2} p^{k}(x) \mathbb{E}_{\epsilon}\left[\left.\frac{\partial^{2} f}{\partial y^{2}}\right|_{y_{\eta}}\right] \mathrm{d} x \\
& =-\left.p^{k}(x) \delta y(x)\right|_{a} ^{b}+\int_{a}^{b} \delta y(x) p^{k \prime}(x) \mathrm{d} x+\int_{a}^{b} \delta y(x) p^{k}(x) \mathbb{E}_{\epsilon}\left[\left.\frac{\partial f}{\partial y}\right|_{y^{\epsilon}}\right] \mathrm{d} x \\
& +\int_{a}^{b} \frac{\delta y(x)^{2}}{2} p^{k}(x) \mathbb{E}_{\epsilon}\left[\left.\frac{\partial^{2} f}{\partial y^{2}}\right|_{y_{\eta}}\right] \mathrm{d} x .
\end{aligned}
$$

Next, to this result we add the fourth addend as follows

$$
\begin{aligned}
& -\left.p^{k}(x) \delta y(x)\right|_{a} ^{b}-\int_{a}^{b} \delta y(x) \delta p^{\prime}(x) \mathrm{d} x+\int_{a}^{b} \delta y(x) \delta p(x) \mathbb{E}_{\epsilon}\left[\left.\frac{\partial f}{\partial y}\right|_{y^{\epsilon}}\right] \mathrm{d} x \\
& +\int_{a}^{b} \frac{\delta y(x)^{2}}{2} p^{k}(x) \mathbb{E}_{\epsilon}\left[\left.\frac{\partial^{2} f}{\partial y^{2}}\right|_{y_{\eta}}\right] \mathrm{d} x \\
& =-p^{k}(b) \delta y(b)+\int_{a}^{b} \delta y^{\prime}(x) \delta p(x) \mathrm{d} x+\int_{a}^{b} \delta y(x) \delta p(x) \mathbb{E}_{\epsilon}\left[\left.\frac{\partial f}{\partial y}\right|_{y^{\epsilon}}\right] \mathrm{d} x
\end{aligned}
$$




$$
+\int_{a}^{b} \frac{\delta y(x)^{2}}{2} p^{k}(x) \mathbb{E}_{\epsilon}\left[\left.\frac{\partial^{2} f}{\partial y^{2}}\right|_{y_{\eta}}\right] \mathrm{d} x
$$

where we used $\delta y(a)=0$ and $\delta p(b)=0$. This expression represents the recast of the sum of the third, fourth and fifth addends of (6.1). Further, from the terminal condition (3.6), we have

$$
-p^{k}(b) \delta y(b)=\phi\left(y^{\epsilon}(b)\right)-\phi\left(y^{k}(b)\right)-\left.\frac{\delta y^{2}(b)}{2} \frac{\partial^{2} \phi}{\partial y^{2}}\right|_{y_{\zeta}},
$$

for an unknown value $y_{\zeta} \in\left[\min \left(y^{\epsilon}(b), y^{k}(b)\right), \max \left(y^{\epsilon}(b), y^{k}(b)\right)\right]$. The term $\delta y^{\prime}(x)$ is elaborated as follows

$$
\begin{aligned}
& \delta y^{\prime}=\mathbb{E}_{\epsilon}\left[f\left(x, y^{\epsilon}, \cdot\right)\right]-\mathbb{E}_{k}\left[f\left(x, y^{k}, \cdot\right)\right]=\mathbb{E}_{\epsilon}\left[f\left(x, y^{\epsilon}, \cdot\right)\right]-\mathbb{E}_{k}\left[f\left(x, y^{\epsilon}-\delta y, \cdot\right)\right] \\
& =\mathbb{E}_{\epsilon}\left[f\left(x, y^{\epsilon}, \cdot\right)\right]-\mathbb{E}_{k}\left[f\left(x, y^{\epsilon}, \cdot\right)-\left.\delta y \frac{\partial f}{\partial y}\right|_{y_{\omega}}\right]=\mathbb{E}_{\epsilon}\left[f\left(x, y^{\epsilon}, \cdot\right)\right]-\mathbb{E}_{\epsilon}\left[f\left(x, y^{\epsilon}, \cdot\right)\right] \\
& +\int_{K_{a d}} f\left(x, y^{\epsilon}(x), v\right) \delta \nu(v, x) \mathrm{d} v+\delta y(x) \mathbb{E}_{k}\left[\left.\frac{\partial f}{\partial y}\right|_{y_{\omega}}\right]
\end{aligned}
$$

thus

$$
\delta y^{\prime}(x)=\int_{K_{a d}} f\left(x, y^{\epsilon}(x), v\right) \delta \nu(v, x) \mathrm{d} v+\delta y(x) \mathbb{E}_{k}\left[\left.\frac{\partial f}{\partial y}\right|_{y_{\omega}}\right]
$$

for some function $y_{\omega}$.

By substituting the last two results in (6.2) and (6.1), we get

$$
\begin{aligned}
0 \geq & \int_{a}^{b}\left[-\mathcal{H}_{\epsilon}\left(x, y^{k}(x), p^{k}(x), \nu^{k}, \nu^{\epsilon}\right)+\mathcal{H}\left(x, y^{k}(x), p^{k}(x), \nu^{k}\right)\right] \mathrm{d} x \\
& \left.\left.=\int_{a}^{b}\left(\mathbb{E}_{\epsilon}\left[\ell\left(x, y^{\epsilon}, \cdot\right)\right)\right]-\mathbb{E}_{k}\left[\ell\left(x, y^{k}, \cdot\right)\right)\right]\right) \mathrm{d} x+\phi\left(y^{\epsilon}(b)\right)-\phi\left(y^{k}(b)\right)-\left.\frac{\delta y^{2}(b)}{2} \frac{\partial^{2} \phi}{\partial y^{2}}\right|_{y_{\zeta}} \\
& +\epsilon \int_{a}^{b} D\left(\nu^{\epsilon} \| \nu^{k}\right) \mathrm{d} x+\int_{a}^{b} \delta p(x) \int f\left(x, y^{\epsilon}(x), v\right) \delta \nu(v, x) \mathrm{d} v \mathrm{~d} x \\
& +\int_{a}^{b} \delta y(x) \delta p(x) \mathbb{E}_{k}\left[\left.\frac{\partial f}{\partial y}\right|_{y_{\omega}}\right] \mathrm{d} x+\int_{a}^{b} \delta y(x) \delta p(x) \mathbb{E}_{\epsilon}\left[\left.\frac{\partial f}{\partial y}\right|_{y^{\epsilon}}\right] \mathrm{d} x \\
& +\int_{a}^{b} \frac{\delta y(x)^{2}}{2} p^{k}(x) \mathbb{E}_{\epsilon}\left[\left.\frac{\partial^{2} f}{\partial y^{2}}\right|_{y_{\eta}}\right] \mathrm{d} x+\int_{a}^{b} \frac{\delta y(x)^{2}}{2} \mathbb{E}_{\epsilon}\left[\left.\frac{\partial^{2} \ell}{\partial y^{2}}\right|_{y_{\xi}}\right] \mathrm{d} x .
\end{aligned}
$$

Then, from the definition of the cost functional, we have

$$
\begin{aligned}
& J\left(y^{\epsilon}, \nu^{\epsilon}\right)-J\left(y^{k}, \nu^{k}\right) \leq-\epsilon \int_{a}^{b} D\left(\nu^{\epsilon} \| \nu^{k}\right) \mathrm{d} x-\int_{a}^{b} \delta p(x) \int f\left(x, y^{\epsilon}(x), v\right) \delta \nu(v, x) \mathrm{d} v \mathrm{~d} x \\
& -\int_{a}^{b} \delta y(x) \delta p(x) \mathbb{E}_{k}\left[\left.\frac{\partial f}{\partial y}\right|_{y_{\omega}}\right] \mathrm{d} x-\int_{a}^{b} \delta y(x) \delta p(x) \mathbb{E}_{\epsilon}\left[\left.\frac{\partial f}{\partial y}\right|_{y^{\epsilon}}\right] \mathrm{d} x
\end{aligned}
$$




$$
-\int_{a}^{b} \frac{\delta y(x)^{2}}{2} p^{k}(x) \mathbb{E}_{\epsilon}\left[\left.\frac{\partial^{2} f}{\partial y^{2}}\right|_{y_{\eta}}\right] \mathrm{d} x-\int_{a}^{b} \frac{\delta y(x)^{2}}{2} \mathbb{E}_{\epsilon}\left[\left.\frac{\partial^{2} \ell}{\partial y^{2}}\right|_{y_{\xi}}\right] \mathrm{d} x+\left.\frac{\delta y(b)^{2}}{2} \frac{\partial^{2} \phi}{\partial y^{2}}\right|_{y_{\zeta}} .
$$

We notice that from the assumptions on the state and adjoint equations, by using the Grönwall's inequality we have the estimates $\|\delta y\|_{1} \leq C_{1}\|\delta \nu\|_{1,1}$ and $\|\delta p\|_{1} \leq C_{2}\|\delta \nu\|_{1,1}$, where $\|\delta \nu\|_{1,1}=\int_{a}^{b} \int_{K_{a d}}|\delta \nu(v, x)| \mathrm{d} v \mathrm{~d} x$, and $|\delta y(b)| \leq C_{3}\|\delta \nu(b, \cdot)\|_{1} \leq C_{4}\|\delta \nu\|_{1,1}$. Since all the derivatives involved are bounded, we obtain

$$
J\left(y^{\epsilon}, \nu^{\epsilon}\right)-J\left(y^{k}, \nu^{k}\right) \leq-\epsilon \int_{a}^{b} D\left(\nu^{\epsilon} \| \nu^{k}\right) \mathrm{d} x+\mathcal{C}\|\delta \nu\|_{1,1}^{2}
$$

for a positive constant $\mathcal{C}$. For the KL-divergence we have the lower bound estimate

$$
D\left(\nu^{\epsilon} \| \nu^{k}\right) \geq \frac{1}{2 \log (2)}\|\delta \nu(\cdot, x)\|_{1}^{2},
$$

that is related to the Pinsker's inequality [31], thus

$$
\int_{a}^{b} D\left(\nu^{\epsilon} \| \nu^{k}\right) \mathrm{d} x \geq \frac{1}{2 \log (2)} \int_{a}^{b}\|\delta \nu(\cdot, x)\|_{1}^{2} \mathrm{~d} x \geq \frac{1}{4 \log (2)}\|\delta \nu\|_{1,1}^{2}
$$

Finally, we obtain

$$
J\left(y^{\epsilon}, \nu^{\epsilon}\right)-J\left(y^{k}, \nu^{k}\right) \leq\left(-\frac{\epsilon}{4 \log (2)}+\mathcal{C}\right)\|\delta \nu\|_{1,1}^{2}
$$

Thus, we have found $\epsilon^{*}=4 \mathcal{C} \log (2)$ such that the claim of the theorem is proved. Therefore, in a finite number of steps, Algorithm 4.1 that eventually increases the value of $\epsilon$ by a factor $\sigma$ reaches the condition $\epsilon>\epsilon^{*}$ such that the corresponding $\nu^{\epsilon}(v, x)$ and the associated state function $y^{\epsilon}$, result in the required decrease of the value of the cost functional.

\section{NUMERICAL EXPERIMENTS}

In this section, we report results of numerical experiments that validate the ability of the SQH algorithm 4.1, with the KL update (5.8), to solve different relaxed optimal control problems. We refer to this scheme as the SQH-KL method.

In order to keep this section to a reasonable size, we focus on a few problems that should demonstrate the applicability of the SQH-KL method to solve control problems with optimal regular or relaxed controls, the cases of oscillations and concentrations, and bang-bang problems.

Furthermore, in order to show robustness of the proposed method, we choose a unique set of numerical parameters for all problems considered below. Specifically, for the interval $K_{a d}$, we consider a partition with subintervals of size $h=\left|K_{a d}\right| / N_{v}$, where $N_{v}=400$. For the interval $I_{a b}=[a, b]$, we consider a grid with mesh size $\mathrm{d} x=|b-a| / N_{x}$, where $N_{x}=200$. We also choose $\epsilon=100, \sigma=1.1, \zeta=0.9, \eta=10^{-3}$, and $\kappa=10^{-4}$. The $\mathrm{SQH}-\mathrm{KL}$ iteration is always initialized with a $\nu^{0}$ with uniform distribution.

We start our series of experiments considering again Problem 3.2, which was proposed by Young [34] to illustrate the meaning of generalised curves referring to the problem of sailing or skiing. In this case $y_{0}=0$ and $K_{a d}=[-1,1]$. The resulting optimal relaxed control is shown in Figure 5. One can see that the resulting $\nu$ numerically approximates the known solution $\frac{1}{2} \delta(v-1)+\frac{1}{2} \delta(v+1)$. Further, we obtain that the value of the state variable $y$ and of the expected value of the control $u_{\text {mean }}(x):=\int_{K_{a d}} v \nu(v, x) \mathrm{d} v$ are in absolute value 


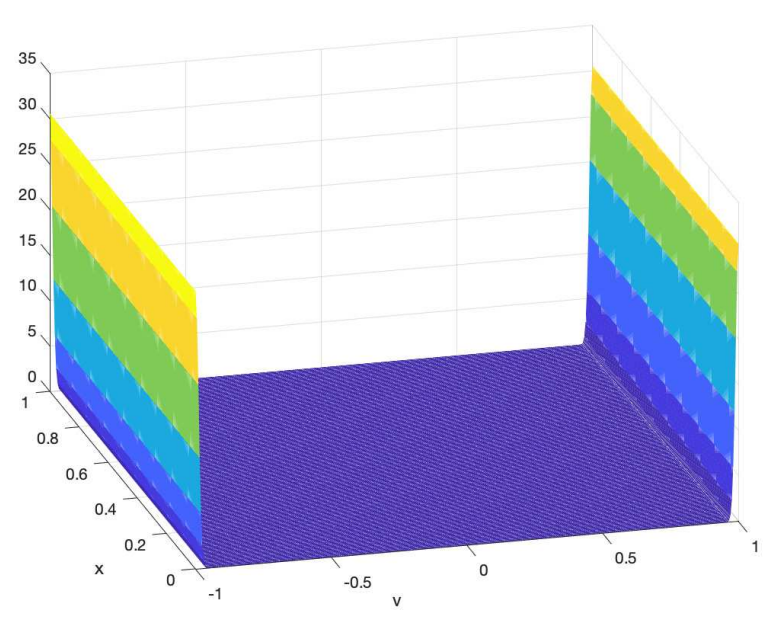

FiguRE 5. Optimal relaxed control for Problem 3.2.

less than $10^{-11}$, that is close to zero machine precision. This experiment illustrates a control problem with oscillations [17].

On the other hand, our framework should be able to solve regular optimal control problems. In order to illustrate this fact, we consider the following tracking problem, where we see that the regular optimal control is obtained as the expected value of the related optimal measure whose variance depends on the weight of the cost of the control.

Problem 7.1. Consider

$$
f(x, y, u)=y+u, \quad \ell(x, y, u)=\left(y-y_{d}\right)^{2}+\alpha u^{2}, \quad \varphi \equiv 0 .
$$

We have $K_{a d}=[-2,2], I_{a b}=[0,1]$ and $y_{0}=1 / 2$. The desired trajectory is given by $y_{d}(x)=\sin (2 \pi x) / 10+1 / 2$, and $\alpha$ is the weight for the cost of the control.

The solution of this problem, obtained with the SQH-KL method, is depicted in Figure 6 with $\alpha=0.01$. In this case, the SQH algorithm terminates with $k=564, J=4.41 \times 10^{-3}, \tau=3.8 \times 10^{-12}$, and $\epsilon=1.2 \times 10^{8}$.

In correspondence to this problem, we report results showing how the value of the weight of the cost $\alpha$ influences the variance of the (numerical) Young measure. Specifically, in Figure 7 we see that larger weights result in smaller variances. Further, notice that with $\alpha=0.1$ the SQH algorithm terminates with $k=366$, $J=26.2 \times 10^{-3}, \tau=0.2 \times 10^{-12}, \epsilon=22.5 \times 10^{8}$; with $\alpha=0.005$ the SQH algorithm terminates with $k=794$, $J=2.58 \times 10^{-3}, \tau=9 \times 10^{-12}, \epsilon=0.53 \times 10^{8}$, and the solution is depicted in Figure 8.

Our next problem is specified as follows.

Problem 7.2. Consider

$$
f(x, y, u)=y+u, \quad \ell(x, y, u)=\left(y-y_{d}\right)^{2}+\left(u^{2}-1\right)^{2}, \quad \varphi \equiv 0 .
$$

We choose $K_{a d}=[-2,2], I_{a b}=[0,1]$ and $y_{0}=1 / 2$. The desired trajectory is given by $y_{d}(x)=\sin (2 \pi x) / 10+1 / 2$.

The solution of Problem 7.2 is depicted in Figure 9. In this case, the SQH-KL algorithm terminates with $k=1745, J=4.37 \times 10^{-4}, \tau=3.9 \times 10^{-14}$, and $\epsilon=9.1 \times 10^{9}$.

Our solution procedure can also be applied to control problems with bang-bang control. To show this fact, we consider the following classical problem of production and consumption; see, e.g., [4] 

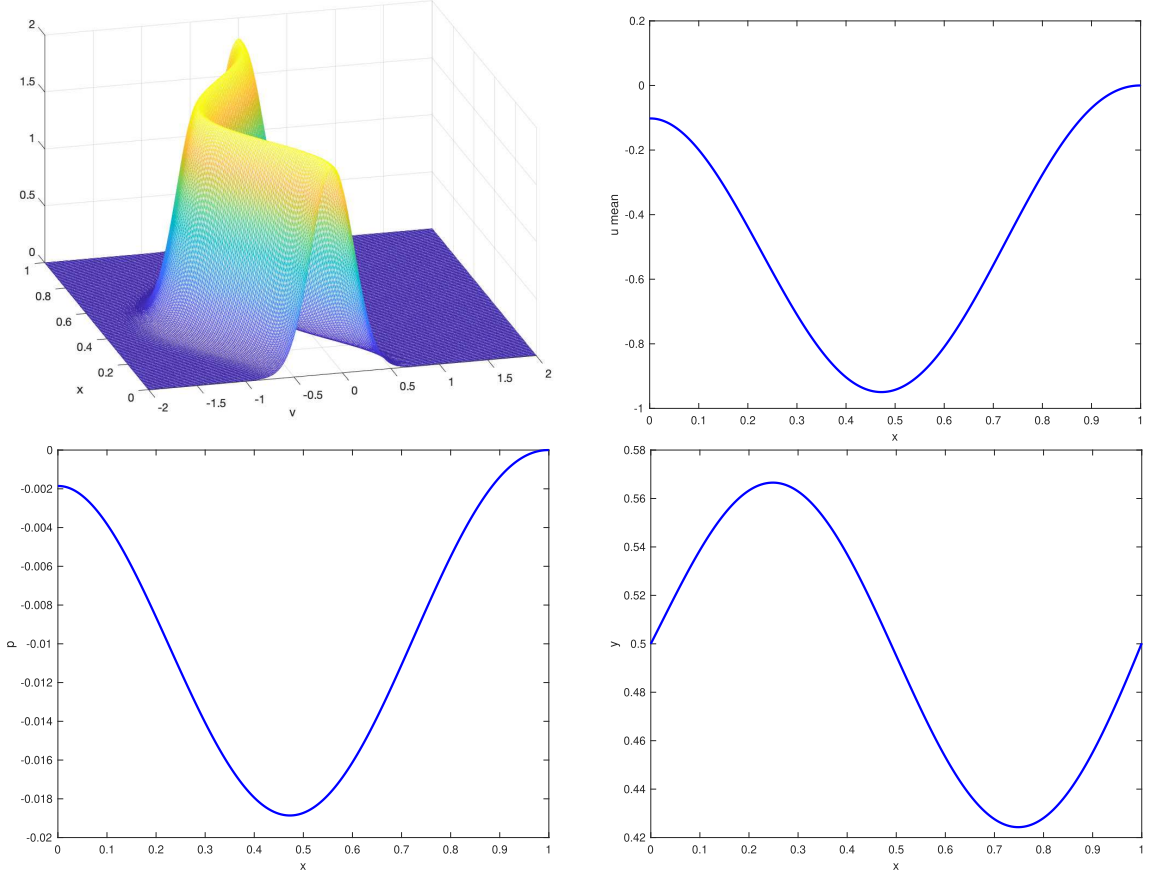

Figure 6. Results for Problem 7.1 with $\alpha=0.01$; the optimal relaxed control and its mean $($ top $)$, and the adjoint and state variables (from left to right).
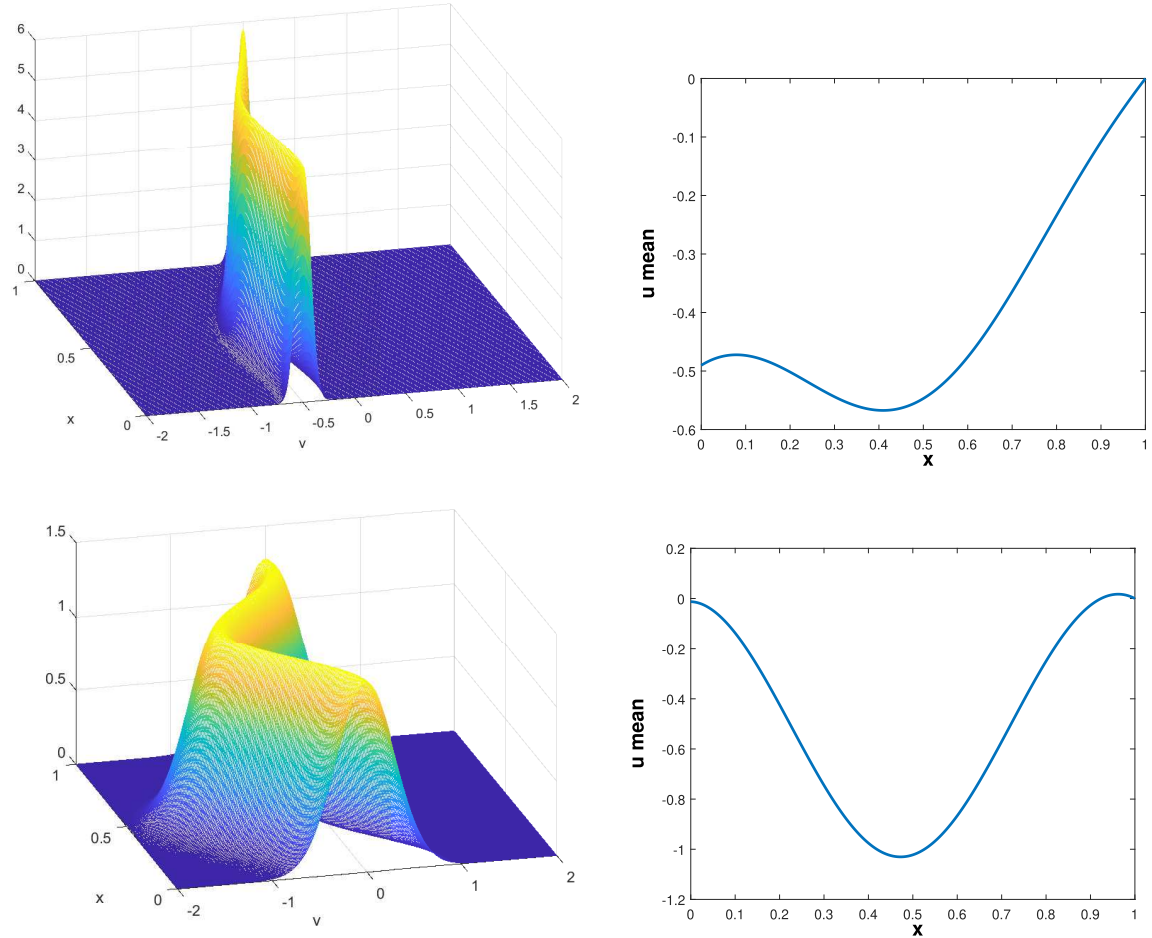

Figure 7. The optimal relaxed control and its mean for Problem 7.1 with $\alpha=10^{-1}$ (top) and $\alpha=5 \times 10^{-3}$ (bottom). 

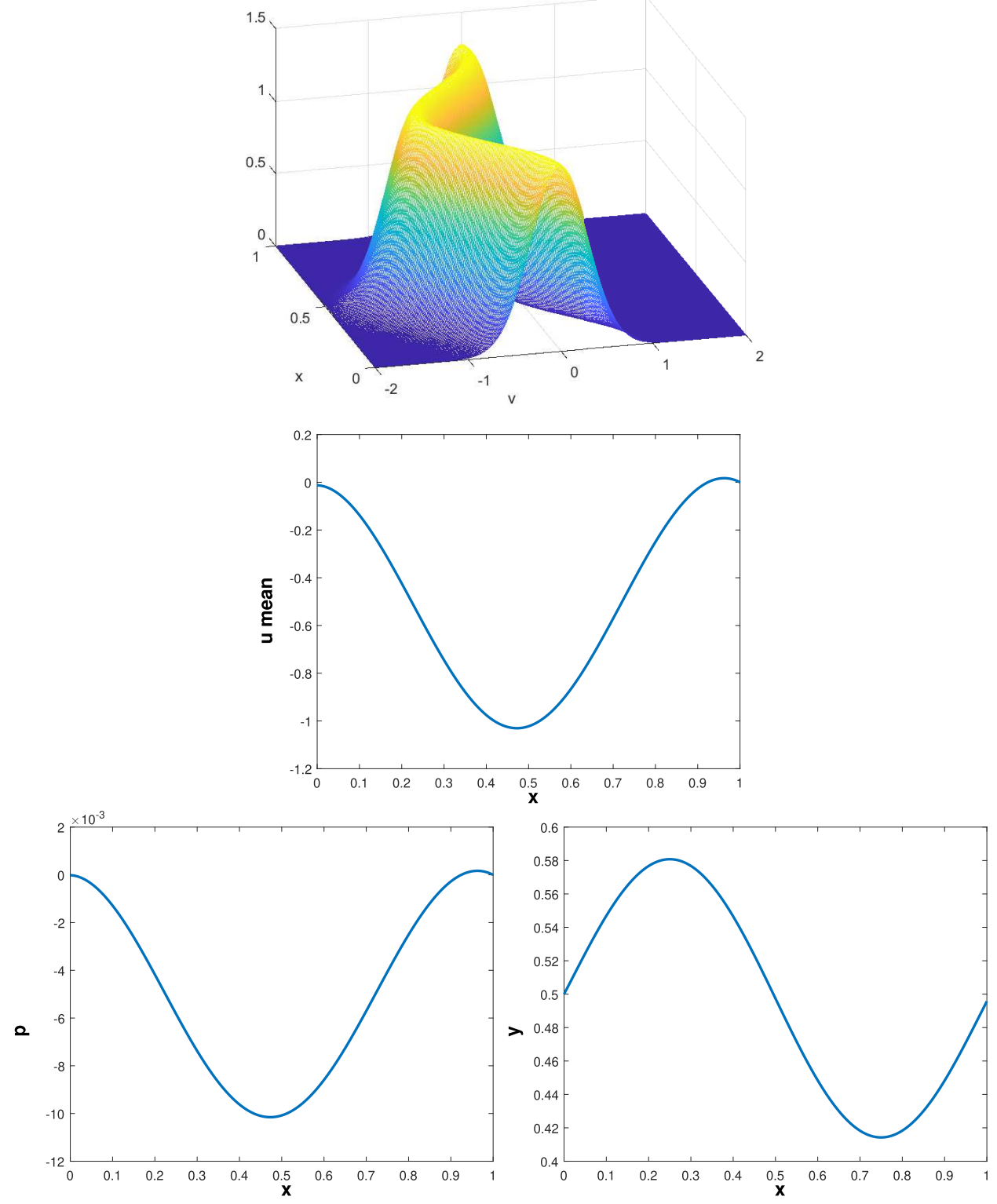

Figure 8. Results for Problem 7.1. $\alpha=5 \times 10^{-3}$.

Problem 7.3. Consider

$$
f(x, y, u)=y u, \quad \ell(x, y, u)=-y(1-u), \quad \varphi \equiv 0 .
$$

We take $K_{a d}=[0,1], I_{a b}=[0,2]$ and $y_{0}=1 / 2$. In this case the control mechanism is bilinear (linear affine) and one can prove that the regular optimal control is the bang-bang control given by

$$
u(x)= \begin{cases}1 & 0 \leq x<1 \\ 0 & 1 \leq x \leq 2\end{cases}
$$



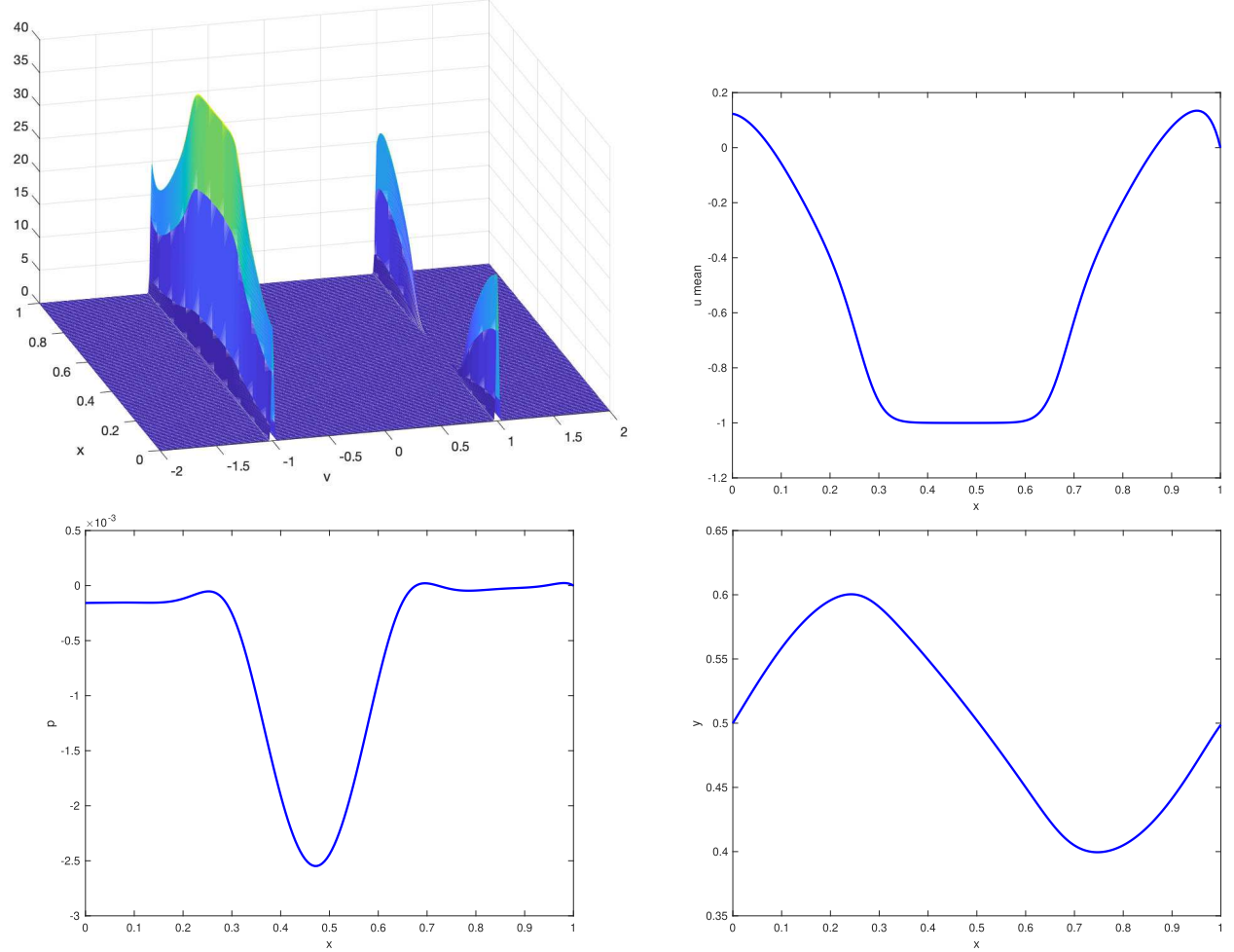

Figure 9. Results for Problem 7.2; the optimal relaxed control and its mean (top), and the adjoint and state variables (from left to right).

We apply the SQH-KL method to solve Problem 7.3 and obtain the results shown in Figure 10. In this case, the SQH-KL algorithm terminates with $k=353, J=-1.365, \tau=1.9 \times 10^{-12}$, and $\epsilon=3.5 \times 10^{8}$.

Next, we consider a problem that involves concentrations effects. See [17] for a similar problem. We have

Problem 7.4. Consider

$$
f(x, y, u)=u, \quad \ell(x, y, u)=u(x-0.5)^{2}, \quad \varphi(y)=(y-1)^{2} .
$$

We choose $K_{a d}=[0,20]$ with the right-hand limit large enough to avoid that this control constraint becomes active. Further, we take $I_{a b}=[0,1]$ and $y_{0}=0$.

The SQH-KL solution to this problem is depicted in Figure 11. In this case, the SQH-KL algorithm terminates with a larger number of iterations $k=4232$, and we obtain $J=4.06 \times 10^{-4}, \tau=4.0 \times 10^{-12}$, and $\epsilon=4.2 \times 10^{7}$.

\section{An APPLICATION WITH TWO RELAXED CONTROLS}

In this section, we consider an application problem with the main purpose of illustrating our approach to solve a relaxed optimal control problem governed by a nonlinear coupled differential system with two controls. Our governing model describes the time evolution of a bioreactor with ideal mixing, where a contaminant and a bacteria that degrades this contaminant are present. This model and a related regular (scalar) optimal control problem are discussed in detail in [24]. In our case, we consider two controls and a different objective functional. 

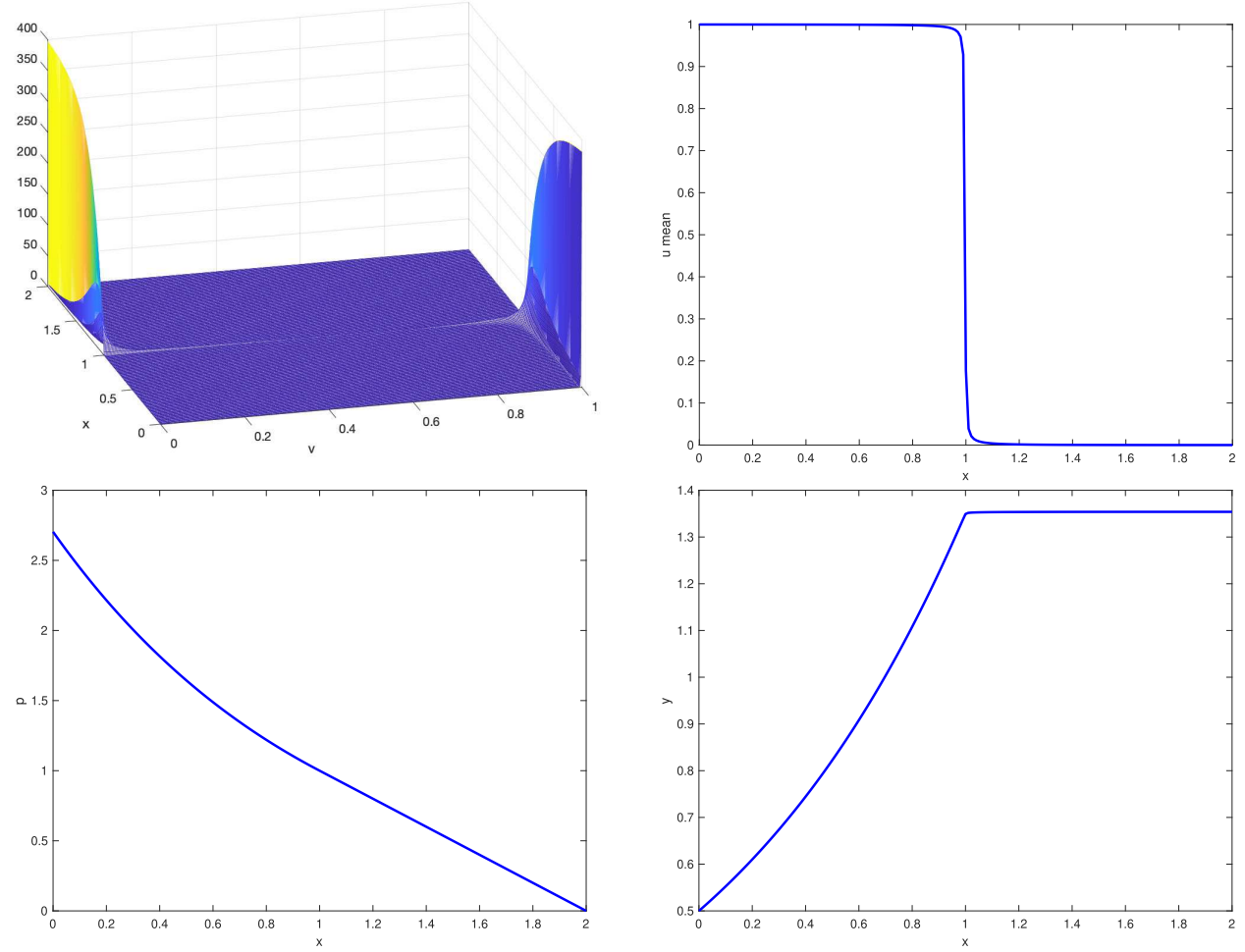

Figure 10. Results for Problem 7.3; the optimal relaxed control and its mean (top), and the adjoint and state variables (from left to right).

Our controlled bioreactor model is given by

$$
\begin{aligned}
& y_{1}^{\prime}(x)=G u_{1}(x) y_{1}(x)-D y_{1}^{2}(x), \\
& y_{2}^{\prime}(x)=-K y_{1}(x) y_{2}(x)+L u_{2}(x),
\end{aligned}
$$

where $x \in[0, T]$, the state variables $y_{1}$ and $y_{2}$ represent, respectively, the concentration of bacteria and contaminant, whereas the controls $u_{1}$ and $u_{2}$ represent nutrient for bacteria and addition of contaminant, respectively.

The constant $G>0$ represents the maximum growth rate of the bacteria, which is modulated by $u_{1}$, while $D>0$ is its death rate. Further, $K>0$ denotes the degradation rate of the contaminant as a by-product of the bacteria metabolism, and $L>0$ is a scaling parameter for the injection of contaminant.

The purpose of the controls is to have the concentration of bacteria follow a given desired profile specified by the function $y_{1}^{d}=y_{1}^{d}(x)$, while at the end of the time horizon the value of the contaminant concentration should be as close as possible to zero. These goals are modelled considering the minimisation of the following objective functional

$$
\begin{aligned}
J\left(y_{1}, y_{2}, \nu^{1}, \nu^{2}\right) & =\frac{1}{2} \int_{0}^{T}\left|y_{1}(x)-y_{1}^{d}(x)\right|^{2} \mathrm{~d} x+\frac{\gamma}{2}\left|y_{2}(T)\right|^{2} \\
& +\alpha \int_{0}^{T} \int_{K_{a d}}\left[\left(\left|v_{1}\left(1-v_{1}\right)\right| \mathrm{d} \nu_{x}^{1}\left(v_{1}\right)\right] \mathrm{d} x\right. \\
& +\beta \int_{0}^{T} \int_{K_{a d}}\left[\left|v_{2}\left(1-v_{2}\right)\right| \mathrm{d} \nu_{x}^{2}\left(v_{2}\right)\right] \mathrm{d} x,
\end{aligned}
$$



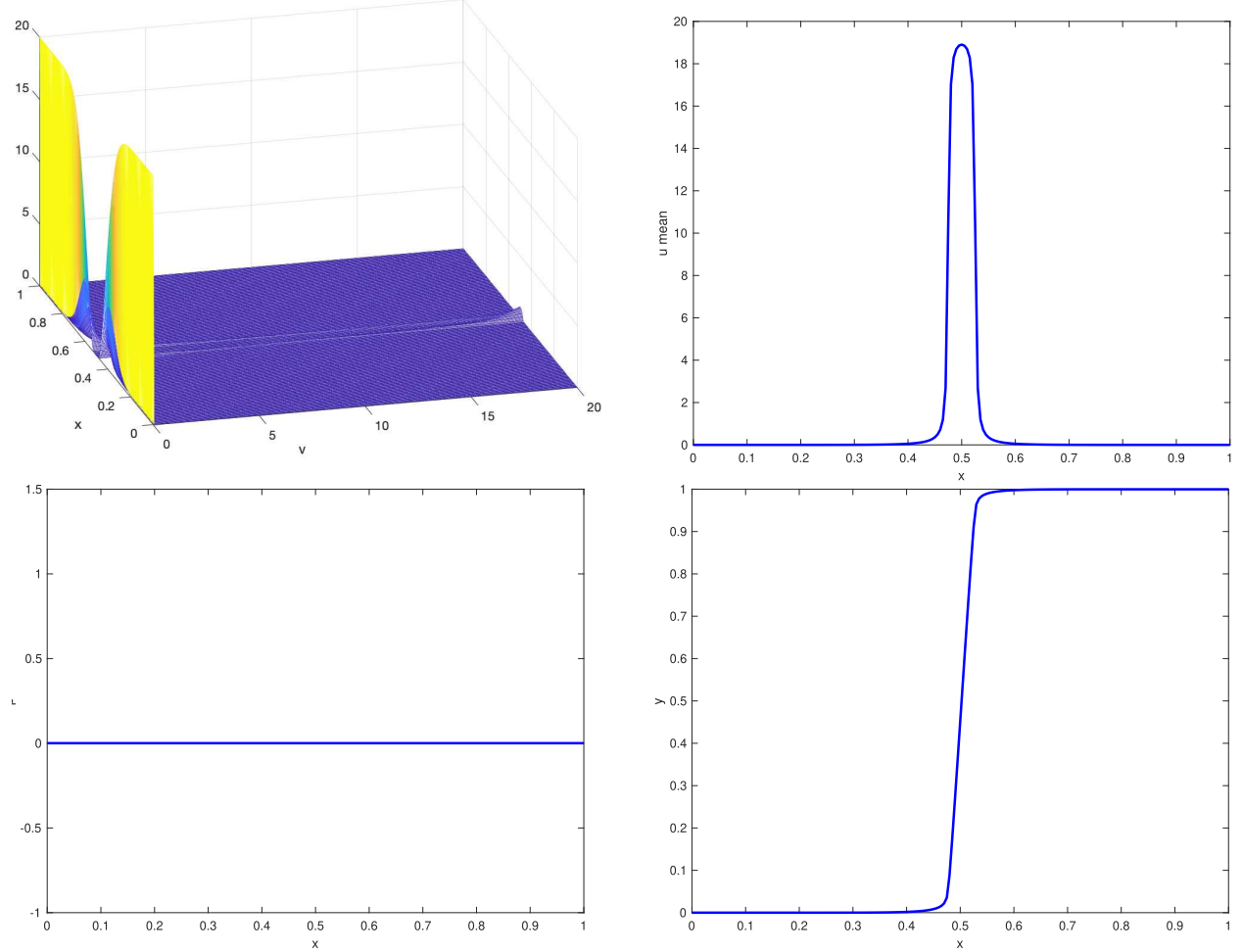

Figure 11. Results for Problem 7.4; the optimal relaxed control and its mean (top), and the adjoint and state variables (from left to right).

where $\alpha, \beta, \gamma>0$ are optimisation weights. For simplicity, we assume that both measures $\nu^{1}$ and $\nu^{2}$, associated to $u_{1}$ and $u_{2}$, are defined on the same $K_{a d}$.

With this setting, the state of the system is computed with (8.1)-(8.2), where $u_{j}(x):=\int_{K_{a d}} v \nu_{x}^{j}(v) \mathrm{d} v$, $j=1,2$, and we have the following adjoint equations

$$
\begin{aligned}
& p_{1}^{\prime}(x)=-G u_{1}(x) p_{1}(x)+2 D y_{1}(x)+K y_{2}(x) p_{2}(x)+\left(y_{1}-y_{1}^{d}\right) \\
& p_{2}^{\prime}(x)=K y_{1}(x) p_{2}(x)
\end{aligned}
$$

with terminal conditions $p_{1}(T)=0$ and $p_{2}(T)=-\gamma y_{2}(T)$.

In the SQH-KL scheme, all parameters are set as specified at the beginning of this section. Clearly, in this case, the augmented Hamiltonian has two augmentation terms as follows

$$
\begin{aligned}
& \mathcal{H}_{\epsilon}\left(x, y_{1}(x), y_{2}(x), p_{1}(x), p_{2}(x), \mu_{x}^{1}, \mu_{x}^{2}, \nu_{x}^{1}, \nu_{x}^{2}\right) \\
& \quad=\mathcal{H}\left(x, y_{1}(x), y_{2}(x), p_{1}(x), p_{2}(x), \nu_{x}^{1}, \nu_{x}^{2}\right)-\epsilon D_{x}\left(\nu^{1} \| \mu^{1}\right)-\epsilon D_{x}\left(\nu^{2} \| \mu^{2}\right) .
\end{aligned}
$$

Now, we notice that our HP function has a composite structure with respect to $\nu^{1}$ and $\nu^{2}$, that is,

$$
\begin{aligned}
\mathcal{H}\left(x, y_{1}(x), y_{2}(x), p_{1}(x), p_{2}(x), \nu_{x}^{1}, \nu_{x}^{2}\right) & =\mathcal{H}^{1}\left(x, y_{1}(x), y_{2}(x), p_{1}(x), p_{2}(x), \nu_{x}^{1}\right) \\
& +\mathcal{H}^{2}\left(x, y_{1}(x), y_{2}(x), p_{1}(x), p_{2}(x), \nu_{x}^{2}\right),
\end{aligned}
$$



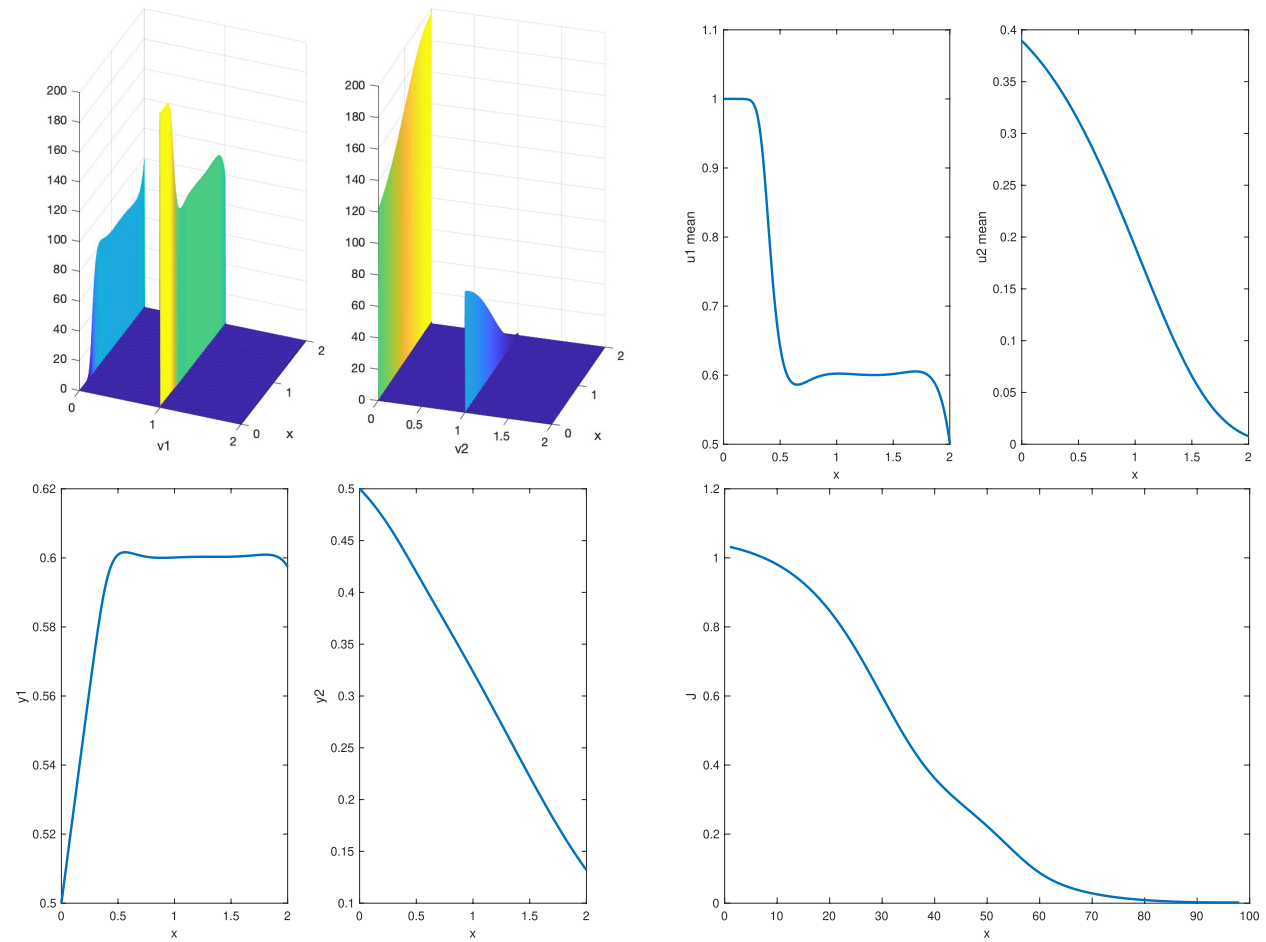

FiguRE 12. Results for a bioreactor problem; the optimal relaxed controls and their mean $(t o p)$; the state variables and the minimisation history of the objective functional (from left to right).

and this is also true for $\mathcal{H}_{\epsilon}$. Therefore the update defined in (5.8) can be applied separately to each measure as follows

$$
\nu^{j}(v)=C_{j} \mu^{j}(v) \exp \left(H^{j}\left(x, y_{1}(x), y_{2}(x), p_{1}(x), p_{2}(x), v\right) / \epsilon\right), \quad j=1,2 .
$$

Further, in Step 3 of the SQH-KL scheme we set

$\tau:=\max \left\{\left\|\nu^{1}-\nu^{1, k}\right\|_{L^{1}\left([a, b] \times K_{a d}\right)},\left\|\nu^{2}-\nu^{2, k}\right\|_{L^{1}\left([a, b] \times K_{a d}\right)}\right\}$, where $\nu^{j, k}, j=1,2$, represent the approximation at the $k$ th iterate of the measures sought.

Next, we report results of an experiment with the following setting: $G=1, D=1, K=2, L=1, \alpha=0.5$, $\beta=0.5, \gamma=0.01$. We require that $y_{1}^{d}(x)=0.6$ is a constant function. Further, we choose $K_{a d}=[0,2], T=2$, and the initial conditions for the state of the bioreactor are given by $y_{1}(0)=0.5$ and $y_{2}(0)=0.5$. The resulting optimal relaxed controls, the time evolution of the controlled system, and the minimisation history of $J$ by SQH iterations are reported in Figure 12.

\section{Conclusion}

This work was devoted to the formulation, analysis, and validation of a sequential quadratic Hamiltonian (SQH) scheme for computing optimal relaxed controls for optimal control problems governed by ordinary differential equations. The $\mathrm{SQH}$ method is an iterative procedure that belongs to the class of successive approximations methods for computing regular controls. Similarly, the working principle of the SQH scheme relies on the characterisation of optimal controls by means of the Pontryagin maximum principle in the framework of Young 
measures. Also distinctive of the proposed SQH scheme is the fact that the relaxed Hamiltonian was augmented by a Kullback-Leibler (KL) divergence term.

The ability of the SQH-KL scheme to solve optimal control problems with relaxed controls, including cases with oscillations and concentration effects, was supported by a theoretical investigation of the SQH-KL minimizing step, and demonstrated by results of numerical experiments.

The formulation of the SQH-KL scheme did not pose any structural restriction to the form of the running costs nor to the dynamics of the governing model. This fact let the authors hope that this methodology may contribute to further investigation of relaxed optimal control problems and to enlarge the range of applicability of this framework.

Acknowledgements. We are very grateful to Emmanuel Trélat and to the two anonymous Referees for comments and suggestions that helped improving this work.

\section{REFERENCES}

[1] J.M. Ball, A version of the fundamental theorem for Young measures. In M. Rascle, D. Serre and M. Slemrod, editors, PDEs and Continuum Models of Phase Transitions. Springer Berlin Heidelberg (1989) 207-215.

[2] V.G. Boltyanskiı̌, R.V. Gamkrelidze and L.S. Pontryagin, On the theory of optimal processes. Dokl. Akad. Nauk SSSR (N.S.) 110 (1956) $7-10$.

[3] J.F. Bonnans, On an algorithm for optimal control using Pontryagin's maximum principle. SIAM J. Control Optim. 24 (1986) $579-588$.

[4] A. Borzì, Modelling with Ordinary Differential Equations: A Comprehensive Approach. Chapman \& Hall/CRC, Abingdon and Boca Raton (2020).

[5] T. Breitenbach and A. Borzì, A sequential quadratic Hamiltonian scheme for solving non-smooth quantum control problems with sparsity. J. Comput. Appl. Math. 369 (2020) 112583.

[6] T. Breitenbach and A. Borzì, On the SQH scheme to solve nonsmooth PDE optimal control problems. Numer. Funct. Anal. Optim. 40 (2019) 1489-1531.

[7] T. Breitenbach and A. Borzì, A sequential quadratic Hamiltonian method for solving parabolic optimal control problems with discontinuous cost functionals. J. Dyn. Control Syst. 25 (2019) 403-435.

[8] C. Castaing, P. Raynaud de Fitte and M. Valadier, Young Measures on Topological Spaces: With Applications in Control Theory and Probability Theory. Springer, Netherlands (2004).

[9] F.L. Chernous'ko and A.A. Lyubushin, Method of successive approximations for solution of optimal control problems. Optim. Control Appl. Methods 3 (1982) 101-114.

[10] M. Claeys, D. Arzelier, D. Henrion and J.-B. Lasserre, Moment LMI approach to LTV impulsive control. In 52nd IEEE Conference on Decision and Control (2013) 5810-5815.

[11] M. Claeys, D. Henrion and M. Kružík, Semi-definite relaxations for optimal control problems with oscillation and concentration effects. ESAIM: COCV 23 (2017) 95-117.

[12] E.A. Coddington and N. Levinson, Theory of Ordinary Differential Equations. Tata McGraw-Hill, New Delhi (1955).

[13] I. Csiszar, I-divergence geometry of probability distributions and minimization problems. Ann. Probab. 3 (1975) 146-158.

[14] B. Dacorogna, Direct Methods in the Calculus of Variations. Springer, New York (2007).

[15] H.O. Fattorini, Infinite Dimensional Optimization and Control Theory. Cambridge University Press, New York (1999).

[16] M. Hale, Y. Wardi, H. Jaleel and M. Egerstedt, Hamiltonian-based algorithm for optimal control. Preprint arXiv:1603.02747 (2016).

[17] D. Henrion, M. Kružík and T. Weisser, Optimal control problems with oscillations, concentrations and discontinuities. Automatica 103 (2019) 159-165.

[18] B. Järmark, A new convergence control technique in differential dynamic programming. Technical Report TRITA-REG-7502, The Royal Institute of Technology, Stockholm, Sweden, Department of Automatic Control (1975).

[19] M. Kružík and T. Roubíček, Optimization problems with concentration and oscillation effects: relaxation theory and numerical approximation. Numer. Funct. Anal. Optim. 20 (1999) 511-530.

[20] I.A. Krylov and F.L. Chernous'ko, On a method of successive approximations for the solution of problems of optimal control. USSR Comput. Math. Math. Phys. 2 (1963) 1371-1382. Transl. of Zh. Vychisl. Mat. Mat. Fiz., 1962, Vol. 2, Nr. 6, $1132-1139$.

[21] I.A. Krylov and F.L. Chernous'ko, An algorithm for the method of successive approximations in optimal control problems. USSR Comput. Math. Math. Phys. 12 (1972) 15-38.

[22] S. Kullback and R.A. Leibler, On information and sufficiency. Ann. Math. Statist. 22 (1951) 79-86.

[23] J.B. Lasserre, D. Henrion, C. Prieur and E. Trélat, Nonlinear optimal control via occupation measures and LMI-relaxations. SIAM J. Control Optim. 47 (2008) 1643-1666.

[24] S. Lenhart and J.T. Workman, Optimal Control Applied to Biological Models. Chapman \& Hall/CRC, Boca Raton (2007).

[25] P. Pedregal, Parametrized Measures and Variational Principles. Progress in nonlinear differential equations and their applications. Birkhäuser (1997). 
[26] L.S. Pontryagin, V.G. Boltyanskiǔ, R.V. Gamkrelidze and E.F. Mishchenko, The Mathematical Theory of Optimal Processes. John Wiley \& Sons, New York-London (1962).

[27] R.T. Rockafellar, Monotone operators and the proximal point algorithm. SIAM J. Control Optim. 14 (1976) 877-898.

[28] T. Roubiček, Relaxation in Optimization Theory and Variational Calculus. De Gruyter, Berlin and New York (1997).

[29] Y. Sakawa and Y. Shindo, On global convergence of an algorithm for optimal control. IEEE Trans. Autom. Control 25 (1980) 1149-1153.

[30] Y. Shindo and Y. Sakawa, Local convergence of an algorithm for solving optimal control problems. J. Optim. Theory Appl. 46 (1985) 265-293.

[31] J. Thomas and T.M. Cover, Elements of Information Theory. Wiley-Interscience (2012).

[32] Y. Wardi, M. Egerstedt and M. Qureshi, Hamiltonian-based algorithm for relaxed optimal control. In 55nd IEEE Conference on Decision and Control (2016) 7222-7227.

[33] J. Warga, Optimal Control of Differential and Functional Equations. Academic Press, New York (1972).

[34] L.C. Young, Lectures on the Calculus of Variations and Optimal Control Theory. W.B. Saunders Company, Philadelphia (1969). 